\title{
Fc-optimized antibodies elicit CD8 immunity to viral respiratory infection
}

https://doi.org/10.1038/s41586-020-2838-z

Received: 18 April 2020

Accepted: 2 October 2020

Published online: 8 October 2020

Check for updates
Stylianos Bournazos ${ }^{1}$, Davide Corti ${ }^{2}$, Herbert W. Virgin ${ }^{3}$ \& Jeffrey V. Ravetch ${ }^{1 凶}$

Antibodies against viral pathogens represent promising therapeutic agents for the control of infection, and their antiviral efficacy has been shown to require the coordinated function of both the Fab and Fc domains ${ }^{1}$. The Fc domain engages a wide spectrum of receptors on discrete cells of the immune system to trigger the clearance of viruses and subsequent killing of infected cells ${ }^{1-4}$. Here we report that Fc engineering of anti-influenza IgG monoclonal antibodies for selective binding to the activating Fc $\gamma$ receptor Fc $\gamma$ RIIa results in enhanced ability to prevent or treat lethal viral respiratory infection in mice, with increased maturation of dendritic cells and the induction of protective $\mathrm{CD}^{+} \mathrm{T}$ cell responses. These findings highlight the capacity for IgG antibodies to induce protective adaptive immunity to viral infection when they selectively activate a dendritic cell and $\mathrm{T}$ cell pathway, with important implications for the development of therapeutic antibodies with improved antiviral efficacy against viral respiratory pathogens.
Several monoclonal antibodies to influenza virus epitopes from the globular head and the stalk domains of influenza hemagglutinin (HA) and neuraminidase (NA) (Fig. 1a) have been shown to confer broad and potent antiviral activity against diverse influenza strains ${ }^{5-8}$. These broadly protective monoclonal antibodies require Fc effector activity to provide full protection from lethal viral challenge, as mutant Fc domain variants that are unable to interact with Fc receptors (Fc $\gamma R s$ ) expressed on effector leukocytes are associated with reduced in vivo antiviral potency ${ }^{5,6}$. Although previous studies clearly demonstrated that broadly protective anti-influenza monoclonal antibodies depend on activating, but not inhibitory, Fc $\gamma$ Rs for activity ${ }^{5,6}$, the cell types and specific Fc $\gamma$ Rs that contribute to the antiviral activity of these monoclonal antibodies remains to be determined. The diversity of Fc $\gamma$ R expression on immune cells, the structural complexity of the Fc $\gamma \mathrm{R}$ family, and the divergence of these receptors in different species (reviewed in ref. ${ }^{9}$ ) pose particular challenges in resolving the mechanistic details of how Fc $\gamma$ R dependence of anti-influenza antibodies result in enhanced protection in vivo.

To address this problem, we previously described a mouse model in which only human $\mathrm{Fc} \gamma \mathrm{Rs}$ are expressed in a pattern that recapitulates the expression pattern seen in human tissues as accurately as possible ${ }^{10}$. Despite the inherent limitations of the use of animal models to study human infection and disease, as well as differences in the absolute FcyR expression levels and pattern of expression between humans and Fc $\gamma \mathrm{R}$-humanized mice (for example, the expression of Fc $\gamma \mathrm{RIIb}$ on monocytes and neutrophils that are commonly absent in humans), this strain represents a suitable platform to perform studies on human IgG antibody function with translational relevance to humans. This in vivo system is combined with anti-influenza antibodies (Fig. 1a) in which the human IgG1 Fc is expressed as a series of variants with selective binding affinity to specific human FcyRs (Fig. 1b, Extended Data Fig. 1a-j). These antibodies are administered to Fc $\gamma \mathrm{R}$-humanized mice before lethal challenge with influenza virus (intranasally, with five mouse lethal doses at $\left.50 \%\left(\mathrm{mLD}_{50}\right)\right)$ and weight loss and survival were monitored over 14 days. Mice treated with broadly protective monoclonal antibodies that target the stalk domain of $\mathrm{HA}\left(\mathrm{FI} 6 \mathrm{~V} 3^{8}\right.$ or FY1 $^{7}$ ) showed enhanced protection when the Fc is modified to selectively engage the Fc $\gamma$ RIIa receptor (GA variant ${ }^{11,12}$ ), alone or in combination with enhanced Fc $\mathrm{R}$ RIIIa binding (GAALIE variant ${ }^{13}$ ) (Fig. 1c, d). Although FcyRIla-enhanced variants (GA) of FY1 fully protect mice that express only human Fc $\gamma$ RIIa under the conditions tested, they are unable to confer antiviral protection of Fc $\gamma \mathrm{R}$-deficient mice, confirming the dependence on Fc $\gamma$ RIIa engagement in the enhanced protection mediated by GA variants (Extended Data Fig. 5a-d). Similarly, the administration of a blocking monoclonal antibody with relative selectivity against Fc $\gamma$ RIIa (clone IV.3) reduces the ability of Fc $\gamma$ RIIa-enhanced variants (GA) of FI6v3 to protect Fc $\gamma \mathrm{R}$-humanized mice against lethal influenza challenge (Extended Data Fig. 5e,f). Enhancing Fc $\gamma$ RIIIa binding aloneusing two complementary approaches: (i) protein engineering (ALIE variant ${ }^{12,14}$ ) or (ii) glycoengineering (afucosylated glycoforms $\mathrm{s}^{15}$ ) - does not provide enhanced protection compared with the wild-type human IgG1 at the selected monoclonal antibody dose (determined based on titration studies that established the antibody dose at which wild-type IgG1 offers sub-optimal protection) (Extended Data Fig. 2). Consistent with previous studies that determined a key role for activating Fc $\gamma \mathrm{R}$ engagement in the anti-viral activity of the selected monoclonal antibodies $^{5,6}$, no protection is observed when the Fc is modified to abrogate Fc $\gamma R$ binding (GRLR variant) or engineered to engage the inhibitory Fc RIIb $\left(V 11\right.$ variant $^{16}$ ) (Fig. 1c, d). None of these Fc modifications affect the in vitro neutralization activity and target antigen-binding specificity (Extended Data Fig. 3a-f) or cause protein aggregation and altered pharmacokinetics in vivo (Extended Data Figs. 1c, d, 4a). In addition, quantification of the serum levels of anti-influenza monoclonal antibodies on day 3 after infection revealed comparable levels among the 


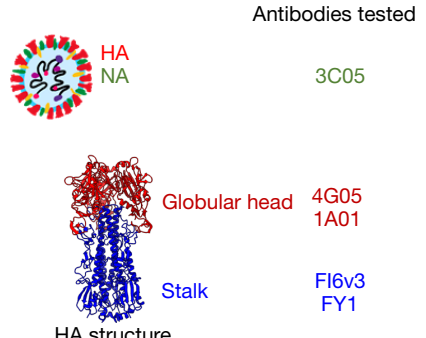

b

\begin{tabular}{|c|c|c|c|c|}
\hline Fc variant & $\mathrm{Fc} \gamma \mathrm{RI}$ & Fc $\gamma$ RIla & FçRIlb & $\mathrm{Fc} \gamma \mathrm{R} I \mathrm{Ila} / \mathrm{b}$ \\
\hline WT & ++++ & ++ & ++ & ++ \\
\hline GRLR & - & - & - & - \\
\hline GA & +++ & +++++ & ++ & ++ \\
\hline ALIE & +++++ & ++ & ++ & +++++ \\
\hline Afuc & ++++ & ++ & ++ & +++++ \\
\hline GAALIE & ++++ & +++++ & + & +++++ \\
\hline V11 & + & + & +++++ & - \\
\hline
\end{tabular}

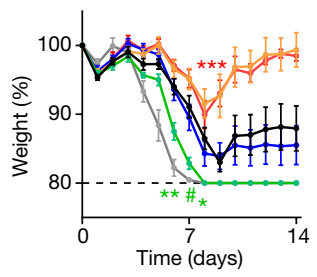

Fl6v3, broadly (group 1 and 2) neutralizing anti-HA stalk

- GAALIE - GA - WT - ALIE - GRLR - PBS

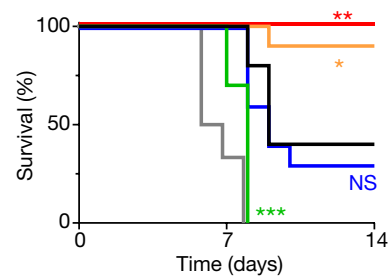

d

FY1, broadly (group 1 and 2) neutralizing anti-HA stalk

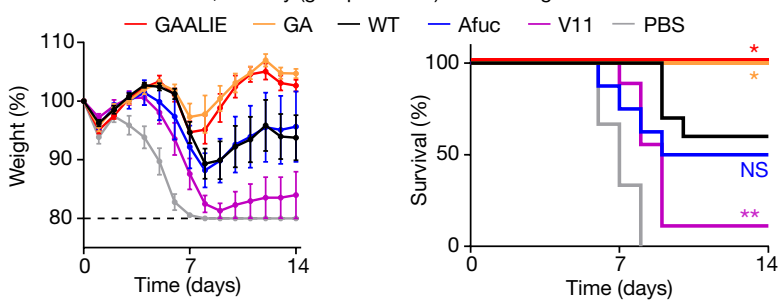

Fig. 1 |Anti-HA stalk monoclonal antibodies engineered for increased FcyRIIa affinity exhibit improved protective activity. a, Influenza virus antigens (HA and NA) and the names of the tested monoclonal antibodies. b, Fc variants with differential Fc $\gamma \mathrm{R}$ binding affinity were generated for anti-influenza monoclonal antibodies. WT, wild type.c, d, Fc variants of anti-HA stalk monoclonal antibodies FI6v3 (c) and FY1 (d) were administered intraperitoneally ( $4 \mathrm{mg} \mathrm{kg}^{-1}$ for FI6v3 and $2 \mathrm{mg} \mathrm{kg}^{-1}$ for FY1) to Fc $\mathrm{R}$-humanized mice before challenge with influenza (H1N1PR8) $(n=6$ mice per group for PBS-treated; $n=10$ for WT FI6v3, GA, ALIE, GRLR and WT FY $1 ; n=9$ for FI6v3
GAALIE and FY1 V11; and $n=8$ for FY1 GA, afucosylated (Afuc), and GAALIE in two independent experiments). Weight loss (left; mean \pm s.e.m.) and survival curves (right) were compared to the corresponding wild-type human IgG1 antibody-treated group by two-way ANOVA (Bonferroni post hoc analysis adjusted for multiple comparisons) $\left(c:{ }^{* *} P=0.0072, \# P=0.0029,{ }^{*} P=0.014\right.$, $\left.{ }^{* * *} P=0.0035\right)$ and log-rank (Mantel-Cox) test, respectively $\left(\mathbf{c}:{ }^{*} P=0.019654\right.$; $\left.{ }^{* *} P=0.006759 ;{ }^{* * *} P=0.000303 ; \mathrm{d}:{ }^{*} P=0.0494,{ }^{* *} P=0.0045\right)$. NS, not significant. different Fc domain variants, which indicates that the observed effects could not be attributed to differential monoclonal antibody half-lives or in vivo stability (Extended Data Fig. $4 \mathrm{~b}, \mathrm{c}$ ).

To determine whether the dependence on Fc $\gamma$ RIIa for the antiviral protection conferred by anti-HA stalk antibodies also extends to antibodies against other viral epitopes, we generated Fc domain variants for the $4 \mathrm{GO5}^{5}$ and $1 \mathrm{AO}^{5}$ antibodies, which target the globular head of $\mathrm{HA}$ and exhibit differential neutralization and haemagglutination inhibition (HAI) activity, as well as for the broadly reactive anti-NA monoclonal antibody, $3 \mathrm{CO}^{17}$ (Extended Data Fig. $3 g-0$ ). Similar to anti-HA stalk antibodies, Fc variants with enhanced affinity for Fc $\gamma$ RIIa (GA or GAALIE variants) demonstrate enhanced protective activity compared with their wild-type human IgG1 counterparts (Fig. 2, Extended Data Fig. $4 d-f$ ), which suggest that the Fc $\gamma R$ mechanisms by which anti-influenza monoclonal antibodies confer protection against infection are conserved among antibodies with differential in vitro neutralization potency and epitope specificity.

Our findings demonstrate that although Fc $\gamma$ RIla is the major receptor that drives the protective activity of anti-influenza monoclonal antibodies, FcyRIIIa has paradoxically limited contribution to the antibody-mediated protection, despite numerous studies that have previously determined that the cytotoxic clearance of malignant or virus-infected cells is predominantly mediated by FcүRIIIa ${ }^{2,12}$. In addition, despite the abundant expression of Fc $\gamma$ RIIIa on alveolar macrophages at baseline as well as the influx of FcyRIIla-expressing natural killer cells in response to infection (Extended Data Fig. 6a-c), selective engagement of this receptor does not enhance protection, suggesting that enhancing the clearance of viral particles by alveolar macrophages or killing of infected cells by natural killer cells does not improve the ability of these antibodies to protect against lethal influenza challenge.

Fc $\gamma$ Rs can either activate (Fc $\gamma$ RI, Fc $\gamma$ RIIa and Fc $\gamma$ RIIIa) or inhibit (Fc $\gamma \mathrm{RIIb}$ ) cellular responses. Activating Fc $\gamma$ Rs trigger intracellular signalling subsequent to crosslinking of the extracellular ligand binding domains by IgG immune complexes by either intrinsic cytoplasmic
ITAM motifs (Fc $\gamma$ RIIa) or $\gamma$ or $\zeta$ chain-associated ITAM motifs (FcyRIIIa), recruiting SYK family tyrosine kinases (reviewed in ref. ${ }^{1}$ ). Because Fc $\gamma$ RIIa and Fc $\gamma$ RIIIa are redundantly expressed on a variety of innate immune cells, including monocytes and macrophages (Extended Data Fig. 6d-f), it is unlikely that the unique dependence on Fc $\gamma$ RIIa engagement that results in enhanced antiviral protection is mediated by these cells. Although neutrophils co-express Fc $\gamma$ RIIa and Fc $\gamma$ RIIIb, the latter lacks intracellular signalling domains and has limited ability to mediate effector activities ${ }^{1}$, even when engaged by variants (ALIE and afucosylated) that exhibit increased affinity for this receptor. It is therefore possible that neutrophils, through specific activation of Fc $\gamma$ RIIa, might contribute to the observed antiviral potency of Fc $\gamma$ RIIa-enhanced variants. To test this hypothesis, we assessed whether or not neutrophil depletion influences the ability of FcyRIIa-enhanced variants (GA) of FY1 to protect Fc $\gamma \mathrm{R}$-humanized mice from lethal influenza challenge. We found that depletion of neutrophils had no effect on the antiviral activity of Fc $\gamma$ RIIa-enhanced FY1 (Extended Data Fig. 5h, i), which suggests that neutrophils are unlikely to contribute notably to the Fc $\gamma$ RIIa-mediated antiviral protection we observe.

In contrast to alveolar macrophages and monocytes, conventional dendritic cells (such as $\mathrm{CDC} 1\left(\mathrm{CD} 11 \mathrm{c}^{+} \mathrm{CD} 11 \mathrm{~b}^{-} \mathrm{CD} 103^{+}\right.$) and $\mathrm{CDC} 2$

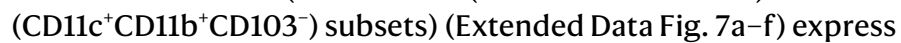
Fc $\gamma$ RIIa and the inhibitory receptor Fc $\gamma$ RIIb, but not Fc $\gamma$ RIIIa, and are found both at baseline and after infection in the lung (Extended Data Fig. 7b). To investigate the effect of FcyRIIa engagement by Fc-engineered monoclonal antibodies on the functional activity of the various dendritic cell subsets (cDC1, cDC2 and Fc $\gamma$ RIII-expressing TNF/iNOS-producing dendritic cells (tipDCs) that emerge in response to influenza infection; defined as $\mathrm{MHCII}^{+} \mathrm{CD} 11 \mathrm{c}^{+} \mathrm{CD} 11 \mathrm{~b}^{+} \mathrm{CD} 103^{-} \mathrm{Gr}-\mathrm{1}^{+}$) (Extended Data Fig. 7a), we analysed dendritic cells in the lungs of Fc $\gamma R$-humanized mice infected with influenza that have previously received Fc variants of the anti-HA stalk monoclonal antibody FI6v3. The treatment of mice with the Fc $\gamma$ RIIa-enhancing variant (GAALIE) before influenza challenge resulted in dendritic cell maturation (Fig. 3a, b, Extended Data Fig. 7g-i, I-n), as seen by the increased frequency of 
a

4G05, broadly (pan-H1) neutralizing anti-HA head
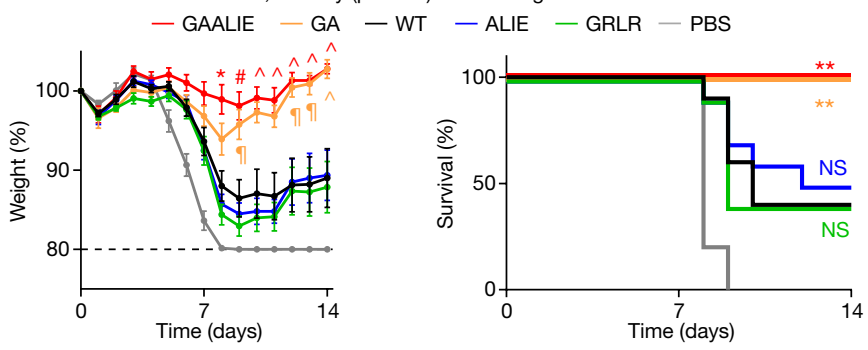

b
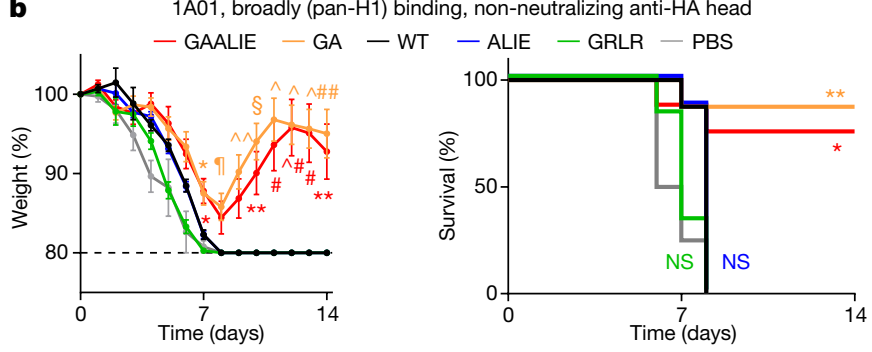

C
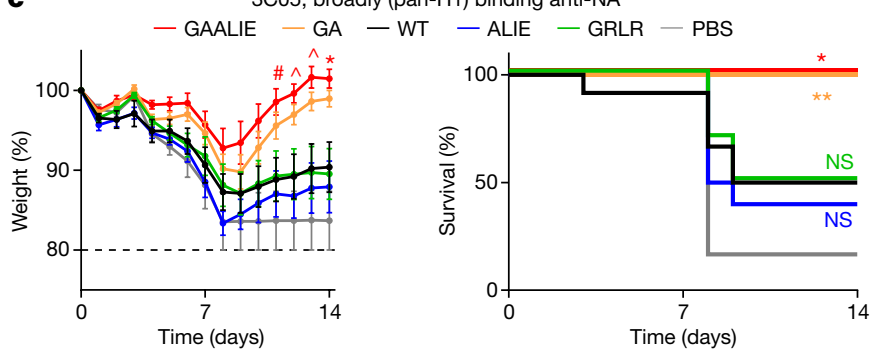

Fig. 2 | Anti-HA globular head and anti-NA antibodies depend on Fc $\gamma$ RIIa to confer protective activity in vivo. a-c, $F c$ variants with differential $F c \gamma R$ affinity (Fig. 1b) were generated for the anti-HA head monoclonal antibodies 4G05 (a) and 1A01 (b), and the anti-NA monoclonal antibody 3C05 (c). Fc $\mathrm{R}^{\mathrm{R}-}$ humanized mice were administered intravenously with Fc domain variants of $4 \mathrm{G} 05\left(0.5 \mathrm{mg} \mathrm{kg}^{-1}\right)(n=10$ mice per group for antibody-treated groups; $n=5$ for PBS group in two independent experiments) (a), $1 \mathrm{A001}\left(2 \mathrm{mg} \mathrm{kg}^{-1}\right)(n=8$ mice per group for WT, GA, ALIE and GAALIE groups; $n=6$ for GRLR; $n=4$ for PBS in two independent experiments) (b), and $3 \mathrm{C} 05\left(20 \mathrm{mg} \mathrm{kg}^{-1}\right)(n=12$ mice per group for WT and GA groups; $n=10$ for GRLR and ALIE; $n=9$ for GAALIE; $n=6$ for PBS in two independent experiments) (c) before lethal challenge with influenza (H1N1 Neth/09). Weight loss (left; mean \pm s.e.m.) and survival curves (right) were compared to the corresponding wild-type unmodified IgG1-treated group by two-way ANOVA (Bonferroni post hoc analysis adjusted for multiple comparisons) $\left(\mathrm{a}:{ }^{*} P=0.0034, \# P=0.0052 ;{ }^{\wedge} P=0.02 ; \boldsymbol{\Phi} P=0.03 ; \mathbf{b}:{ }^{*} P=0.03\right.$, ${ }^{* *} P=0.02, \# P=0.01,{ }^{\wedge} P=0.001, \# \# P=0.005, \S P=0.002,{ }^{\wedge} P=0.006, \uparrow P=0.04$, ${ }^{\wedge} \# P=0.009$; c: ${ }^{*} P=0.03,{ }^{\wedge} P=0.02, \# P=0.04$ ) and log-rank (Mantel-Cox) test, respectively $\left(\mathbf{a}:{ }^{* *} P=0.0042, \mathbf{b}:{ }^{* *} P=0.0032,{ }^{*} P=0.01285, \mathbf{c}:{ }^{*} P=0.0167\right.$, $\left.{ }^{* *} P=0.0055\right)$.

CD80 ${ }^{\text {high }} \mathrm{CD} 86^{\text {high }}$ cells on $\mathrm{CDC} 1$ and $\mathrm{CDC} 2$ subsets, and upregulation of $\mathrm{CD} 40$ expression in the $\mathrm{CDC} 1$ subset-the dendritic cell population specialized for cross-presentation and CD8 T cell stimulation ${ }^{18}$. By contrast, the same antibody (FI6v3) expressed either as wild-type or with an Fc modified to abrogate Fc $\gamma$ R binding (GRLR) did not result in dendritic cell maturation. Unlike the $\mathrm{CDC} 1$ and $\mathrm{CDC} 2$ subsets, treatment with the GAALIE variant is not associated with increased maturation of tipDCs, which suggests a limited role for this population in the antibody-mediated protection against influenza infection conferred by FcyRIla-enhanced variants (Extended Data Fig. 7i). Consistent with these in vivo data from Fc $\gamma \mathrm{R}$-humanized mice, ex vivo stimulation of human monocyte-derived dendritic cells with IgG immune complexes revealed improved capacity for Fc $\gamma$ RIla-enhanced variants (GA and GAALIE) to induce cellular maturation (Extended Data Fig. 7j, k).
Maturation of dendritic cells and the induction of the accessory molecules CD80, CD86 and CD40 in the virally challenged lung is a prerequisite to the activation of antigen-specific naive $T$ cells. This would indicate that an anti-viral antibody modified to enhance dendritic cell maturation by Fc $\gamma$ RIla engagement can induce an adaptive response that results in the induction of protective T cell immunity. To explore this hypothesis, we characterized the T cell responses in the lungs of Fc $\gamma$ R-humanized mice treated with anti-influenza monoclonal antibodies with selective Fc $\gamma \mathrm{R}$-binding properties before viral challenge. We found that the GAALIE variant induced increased activation of both $\mathrm{CD}^{+}$and $\mathrm{CD}^{+}{ }^{+} \mathrm{T}$ cells (Fig. 3c, Extended Data Fig. 8), whereas neither wild-type IgG1 nor the GRLR variant showed evidence of robust induction of T cell responses. To determine whether the observed induction of $\mathrm{T}$ cell activation also contributes to the enhanced protection we observed with the Fc $\gamma$ RIIa-binding variants (Figs. 1, 2), we repeated the FY1 antibody pre-treatment and viral challenge protocol, modifying it to include a $\mathrm{CD}^{+}$or $\mathrm{CD}^{+}$cellular depletion step on day 3 after infection (Fig. 3d, Extended Data Fig. 10a-c). Depletion of $\mathrm{CD}^{+}$, but not $\mathrm{CD}^{+}, \mathrm{T}$ cells resulted in the loss of enhancement of the GA or GAALIE Fc variant, demonstrating that the improved protection observed for Fc $\gamma$ RIIa-enhanced variants is mediated by the induction of protective $\mathrm{CD}^{+} \mathrm{T}$ cell responses (Fig. 3e, f, Extended Data Figs. 4g, 10a-e).

Because recent reports have suggested Fc $\gamma$ RIIa expression by subsets of $\mathrm{T}$ cells ${ }^{19-21}$, we analysed $\mathrm{Fc} \gamma \mathrm{R}$ expression in human peripheral T cells, as well as in T cells obtained from the blood, spleen or lung of naive or influenza-infected FcyR-humanized mice (Extended Data Fig. 9) to determine whether the improved protective activity of FcyRIla-enhanced variants is the outcome of direct $T$ cell activation by Fc $\gamma$ R crosslinking. With the exception of a minor (10\%) subset of CD8 ${ }^{+}$ T cells in the lung of infected mice that is positive for Fc $\gamma$ RIII (Extended Data Fig. 9l), T cells from human or Fc $\gamma$ R-humanized mice were negative for all Fc $\gamma$ Rs, which suggests that the antibody-mediated anti-viral protection is not due to direct effects on T cells.

Because FcyRIla-enhanced variants induce the activation of both $\mathrm{CD}^{+}$and $\mathrm{CD}^{+}{ }^{+} \mathrm{T}$ cells, we tested whether these effects also drive improved antiviral IgG responses. IgG responses were characterized in mice treated with wild-type or GAALIE variants of the non-neutralizing anti-HA monoclonal antibody $1 \mathrm{AO} 1$ at a dose that both variants offer comparable protection. Treatment with either wild-type or Fc $\gamma$ RIla-enhanced variants resulted in robust and comparable IgG responses against $\mathrm{HA}$ and NP from homologous or heterologous virus strains, as well as serum HAI activity (Extended Data Fig. 10f $-\mathrm{m}$ ).

Through interactions with effector leukocytes, antibodies against viral antigens can enhance disease and contribute to specific histopathologic manifestations. Although this phenomenon, termed antibody-dependent enhancement, has been shown specifically for flaviviruses, such as dengue ${ }^{22}$, clinical experience from severe cases of viral respiratory infections, such as influenza and SARS-CoV-2, also raise the possibility that antibodies might have a pathogenic role through uncontrolled or inappropriate amplification of local inflammatory responses. For example, studies during the 2009 influenza pandemic have shown that severe disease was associated with evidence of IgG-mediated inflammation in the lung parenchyma through IgG immune complex and complement deposition to the lung tissue ${ }^{23}$.Similarly, severe cases of COVID-19 disease are often characterized by excessive pro-inflammatory cytokine expression and $\mathrm{T}$ cell responses, as well as by clinical manifestations that resemble secondary haemophagocytic lymphohistiocytosis ${ }^{24}$. Given that Fc-engineered variants with increased affinity for Fc $\gamma$ RIIa can enhance adaptive T cell responses by activation of Fc $\gamma$ RIIa-expressing dendritic cells, it is important to determine whether such variants could also modulate disease pathogenesis through inappropriate amplification of host inflammatory responses that are elicited in response to virus infection. To determine whether Fc $\gamma$ RIIa-enhanced variants could lead to severe disease, we assessed the in vivo activity of these variants in Fc $\gamma \mathrm{R}$-humanized mice 


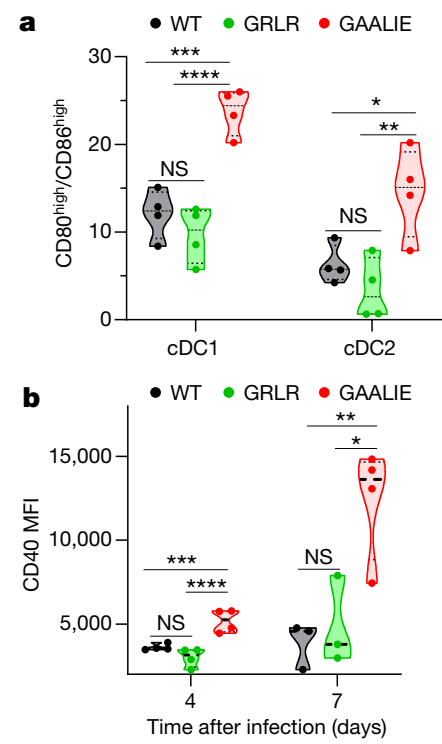

c $\bullet \mathrm{WT} \cdot \mathrm{GRLR} \bullet \mathrm{GAALIE}$

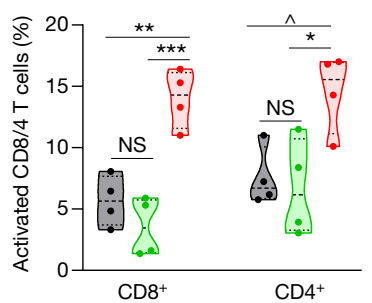

Fig. 3 | Engagement of Fc $\gamma$ RIIa by Fc-engineered monoclonal antibodies drives dendritic cell maturation and protective $\mathrm{CDB}^{+} \mathrm{T}$ cell responses. $\mathrm{a}-\mathrm{c}$, Fc $\gamma$ R-humanized mice ( $n=4$ mice per group for all groups, except $n=3$ for day 7 WT and GRLR) were treated with FI6v3 monoclonal antibody Fc variants (intraperitoneally $3 \mathrm{mg} \mathrm{kg}^{-1}$ ), infected with H1N1 (PR8), and then lung-resident dendritic cells and T cells were analysed on days 4 ( $n=4$ mice per group) or 7 ( $n=3$ mice per group for WT and GRLR, $n=4$ for GAALIE in two independent experiments) after infection. One-way ANOVA (Bonferroni post hoc analysis adjusted for multiple comparisons) was used to compare the frequency of mature conventional dendritic cell subsets $\left(C D 80^{\text {high }} \mathrm{CD} 86^{\text {high }}\right)$ on day 4 after infection $\left({ }^{*} P=0.0384,{ }^{* *} P=0.0075,{ }^{* * *} P=0.0008,{ }^{* * * *} P=0.0002\right)(\mathbf{a}), \mathrm{CD} 40$ expression in $\mathrm{CDC} 1$ subsets $\left({ }^{*} P=0.0261,{ }^{* *} P=0.0139,{ }^{* * *} P=0.0061\right.$, $\left.{ }^{* * * *} P=0.0007\right)(\mathbf{b})$, and the frequency of activated $\left(\mathrm{CD} 44^{\mathrm{hi}} \mathrm{CD} 69^{+}\right) \mathrm{CD} 8^{+}$and

with established influenza infection (Fig. 4a). Mice were infected with influenza and 3 days after infection, FY1 monoclonal antibody (either wild-type or GAALIE) was administered at different doses $\left(5-15 \mathrm{mg} \mathrm{kg}^{-1}\right)$. Although wild-type IgG1 FY1 was unable to rescue mice from lethal influenza infection, GAALIE variants exhibited a dose-dependent therapeutic benefit. These findings suggest that the unique Fc $\gamma R$-binding profile of the GAALIE variant (increased for activating Fc $\gamma$ RIIa and Fc $\gamma$ RIIIla receptors, but reduced for the inhibitory Fc $\gamma$ RIIb receptor), and its ability to stimulate CD8 $\mathrm{T}$ cell responses has no pathogenic consequences, instead it provides meaningful and robust protection from established infection (Fig. 4b, c).

In addition to their therapeutic potential, monoclonal antibodies engineered for increased Fc $\gamma$ RIIa affinity could provide long-term prophylaxis from influenza infection, especially when combined with Fc domain mutations (for example, the LS (M428L/N434S) variant ${ }^{25}$ ) that increase affinity for human FcRn and extend IgG half-life in vivo ${ }^{25}$. Using a mouse model of antibody-mediated prophylaxis of influenza infection (Fig. 4d), we compared the ability of LS (enhanced for FcRn) and GAALIE-LS (enhanced for FcRn, Fc $\gamma$ RIIa and Fc $\gamma$ RIIIa) variants of FY1 to protect Fc $\gamma R$ - or FcRn-humanized mice from influenza infection. At all doses tested $\left(0.1-1.6 \mathrm{mg} \mathrm{kg}^{-1}\right)$, GAALIE-LS variants demonstrated
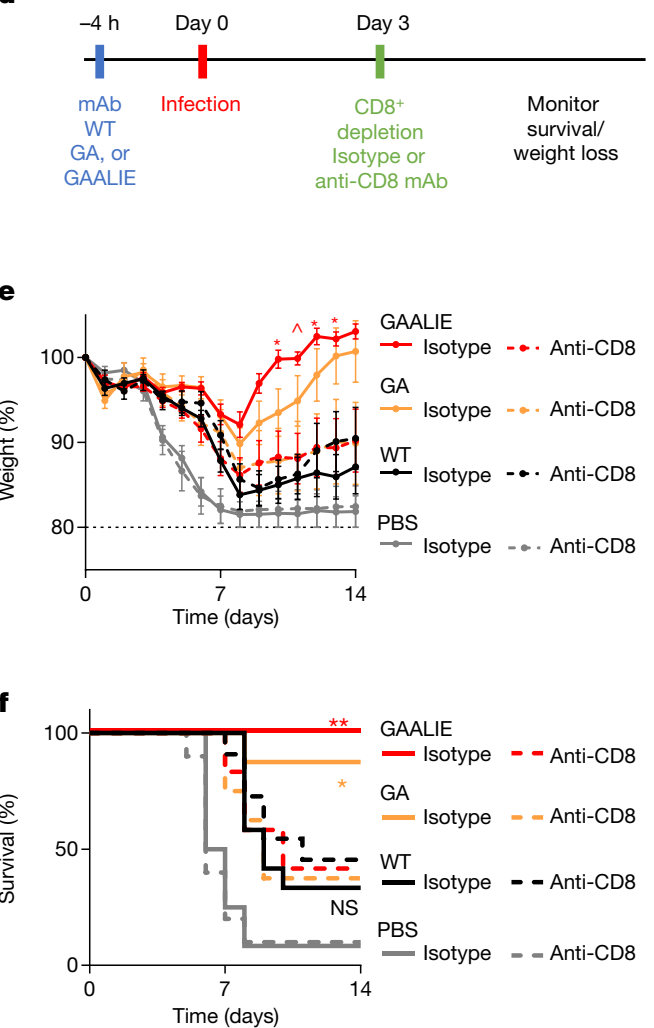

$\mathrm{CD}^{+} \mathrm{T}$ cells (day 4 after infection) $\left({ }^{\wedge} P=0.0412,{ }^{*} P=0.0232,{ }^{* *} P=0.0018\right.$, $\left.{ }^{* * *} P=0.0003\right)(\mathbf{c})$. MFI, mean fluorescence intensity. $\mathbf{d}-\mathbf{f}$, Fc variants of the FY1 monoclonal antibody ( $\mathrm{mAb}$ ) were administered intraperitoneally $\left(2 \mathrm{mg} \mathrm{kg}^{-1}\right)$ to Fc $\gamma R$-humanized mice before influenza challenge (H1N1 PR8). Isotype or anti-mouse CD8 monoclonal antibodies were administered on day 3 after infection (isotype-treated groups: $n=12$ mice per group for WT, GAALIE and PBS groups, $n=8$ mice for GA; anti-CD8-treated groups: $n=11$ mice per group for WT, $n=8$ for GA, $n=10$ for PBS, and $n=12$ for GAALIE in three independent experiments).e,f, Weight loss (mean \pm s.e.m.) (e) and survival curves (f) were compared against the corresponding isotype-treated group by two-way ANOVA (Bonferroni post hoc analysis adjusted for multiple comparisons) $\left({ }^{*} P=0.03, \wedge P=0.0220\right)$ and log-rank (Mantel-Cox) test, respectively $\left({ }^{* *} P=0.00199,{ }^{*} P=0.0477\right)$.

superior protective activity over their LS counterparts (Fig. $4 \mathrm{e}-\mathrm{g}$, Extended Data Fig. $4 \mathrm{~h}$ ). In addition, quantification of the protective activity of the two FY1 Fc variants over a wide range of doses revealed that the GAALIE-LS variant exhibited at least 5.5-fold improvement in in vivo antiviral potency (Fig. $4 \mathrm{~h}$ ), which suggests that Fc engineering for increased affinity to specific $\mathrm{Fc} \gamma \mathrm{Rs}$ represents a promising approach that could substantially improve the clinical efficacy of antiviral monoclonal antibodies.

IgG antibodies can mediate pleiotropic effects, resulting from the diversity of Fc binding molecules that engage the Fc domain. The Fc domain is structurally diverse, the consequence of subclasses and Fc glycosylation, and results in differential Fc receptor binding activities for various Fc structural variants (reviewed in ref. ${ }^{1}$ ). This natural heterogeneity contributes to the efficacy of polyclonal IgG responses to viral infections, and provides a mechanism for the recognition of diverse viral epitopes and triggering of several effector pathways. The development of monoclonal antibodies for the selective binding to specific neutralizing viral epitopes can now be coupled to Fc modifications to facilitate the engagement of specific Fc $\gamma$ Rs to optimize the potency of these therapeutic agents. As demonstrated for anti-tumour therapeutic antibodies, enhancing their affinity for Fc $\gamma$ RIIIa results in improved 


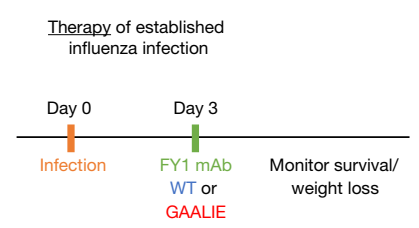

d

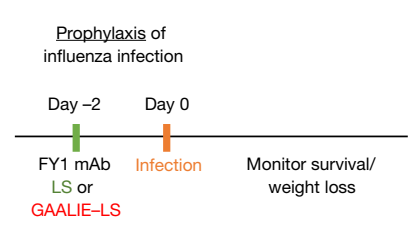

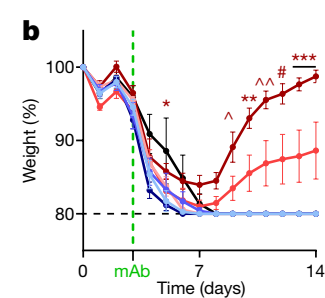

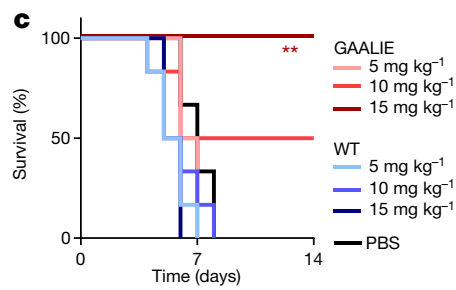

e

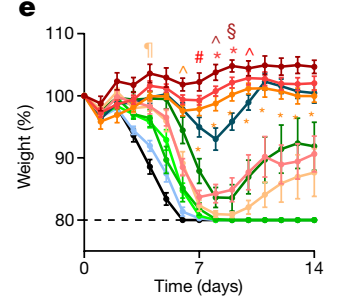

$\mathbf{f}$

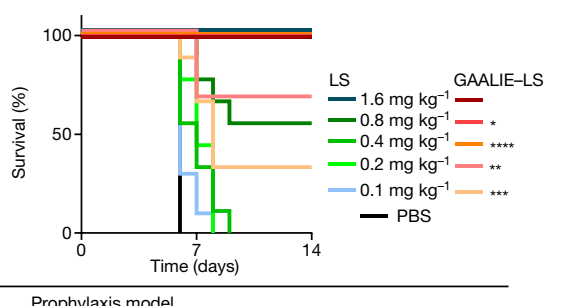

Prophylaxis model

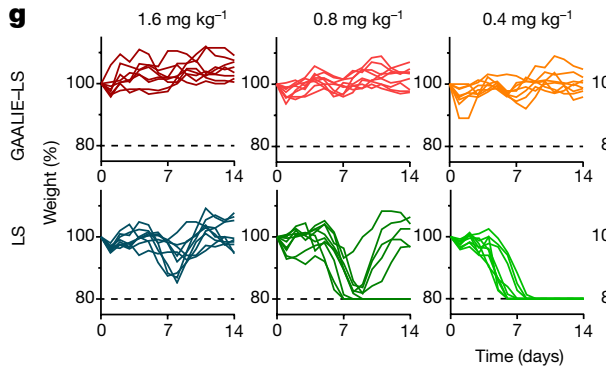

Fig. 4 | Evaluation of GAALIE variants of the FY1 monoclonal antibody in models of therapy or prevention of influenza infection. a-c, Fc $\gamma \mathrm{R}$ humanized mice ( $n=6$ mice per group for antibody-treated groups; $n=3$ for PBS-treated in two independent experiments) were infected with influenza (H1N1 PR8), and FY1 Fc variants were administered intraperitoneally 3 days after infection at the indicated dose. b, c, Weight loss (mean \pm s.e.m.) (b) and survival curves (c) of GAALIE-treated mice were compared with the PBS-treated group at the corresponding antibody dose by two-way ANOVA (Bonferroni post hoc analysis adjusted for multiple comparisons) $\left({ }^{*} P=0.0456,{ }^{\wedge} P=0.041\right.$, $\left.{ }^{* *} P=0.0014,{ }^{\wedge} P=0.0003, \# P=0.0005,{ }^{* * *} P<0.0001\right)$ and log-rank (MantelCox) test, respectively $\left({ }^{* *} P=0.000911\right) . \mathbf{d}-\mathbf{h}$, The protective activity of LS and GAALIE-LS variants of FY1 was evaluated in a model of antibody-mediated prophylaxis. Fc $\gamma \mathrm{R} / \mathrm{FcRn}$-humanized mice ( $n=8$ mice per group for GAALIE-LS

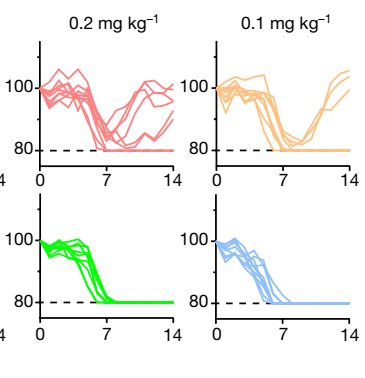

h

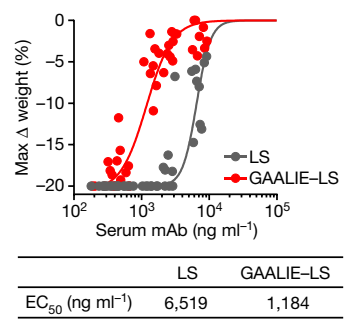

at 1.6 and $0.4 \mathrm{mg} \mathrm{kg}^{-1} ; n=10$ for LS at $0.1 \mathrm{mg} \mathrm{kg}^{-1}, n=3$ for PBS, $n=9$ for the remaining treatment groups in two independent experiments) were administered intravenously with the indicated dose of FY12 days before influenza challenge (H1N1 PR8). e, f, Weight loss (mean \pm s.e.m.) (e) and survival curves (f) of GAALIE-LS-treated mice were compared against the LS group at the corresponding antibody dose by two-way ANOVA (Bonferroni post hoc analysis adjusted for multiple comparisons) $\left({ }^{*} P<0.001,{ }^{\wedge} P=0.01, \# P=0.02\right.$, $\S P=0.006, \Phi P=0.002$ ) and log-rank (Mantel-Cox) test, respectively $\left({ }^{*} P=0.0283,{ }^{* *} P=0.0266,{ }^{* * *} P=0.00377,{ }^{* * * *} P=0.000143\right) . \mathbf{h}$, The enhanced potency conferred by the GAALIE-LS variant was quantified by plotting the maximum weight change after infection against the serum antibody concentration at the time of challenge. Data were fitted by nonlinear regression analysis (four-parameter, variable slope). phagocytosis of tumour cells by macrophages (antibody-dependent cell phagocytosis, ADCP), as well as enhanced killing by natural killer cells (antibody-dependent cell cytotoxicity, ADCC), which translates to improved clinical efficacy ${ }^{26}$. On the basis of these findings, it has been presumed that enhancing the ability of the Fc domain to engage and activate Fc $\gamma$ RIIIla would also lead to increased therapeutic efficacy of protective anti-viral antibodies. However, this does not seem to be the case. Antibody treatment of HIV infection has been shown to induce a $\mathrm{CD}^{+}$response both in chronically infected macaques and humans, which contributes to the control of viraemia ${ }^{27,28}$. Our present results show that selective engagement of the activating $\mathrm{Fc} \gamma \mathrm{R}$ on dendritic cells, Fc $\gamma$ RIIa, by a variety of anti-influenza monoclonal antibodies results in the induction of a protective $\mathrm{CD} 8^{+}$response, mechanistically similar to the 'vaccinal' response we have observed for anti-tumour antibody treatment ${ }^{12}$. The ability of an antibody to not only couple to innate effector responses through its Fc domain, but also induce an adaptive response by engaging and activating dendritic cells, provides a potent new approach to the design of therapeutic antibodies for the prevention and treatment of viral diseases. This approach to Fc engineering is particularly relevant to pandemic viruses, such as influenza and SARS-CoV-2. Neutralizing antibodies to these viruses, engineered to enhance dendritic cell activation and $C D 8^{+}$
T cell responses, as shown here for the GAALIE variant, are predicted to provide considerable enhancement of protection by stimulating a variety of synergistic immunological pathways.

\section{Online content}

Any methods, additional references, Nature Research reporting summaries, source data, extended data, supplementary information, acknowledgements, peer review information; details of author contributions and competing interests; and statements of data and code availability are available at https://doi.org/10.1038/s41586-020-2838-z.

1. Bournazos, S., Wang, T. T., Dahan, R., Maamary, J. \& Ravetch, J. V. Signaling by antibodies: recent progress. Annu. Rev. Immunol. 35, 285-311 (2017).

2. Lu, C. L. et al. Enhanced clearance of HIV-1-infected cells by broadly neutralizing antibodies against HIV-1 in vivo. Science 352, 1001-1004 (2016).

3. Bournazos, S., DiLillo, D. J. \& Ravetch, J. V. The role of Fc-FcyR interactions in IgG-mediated microbial neutralization. J. Exp. Med. 212, 1361-1369 (2015).

4. Bournazos, S. et al. Broadly neutralizing anti-HIV-1 antibodies require Fc effector functions for in vivo activity. Cell 158, 1243-1253 (2014).

5. DiLillo, D. J., Palese, P., Wilson, P. C. \& Ravetch, J. V. Broadly neutralizing anti-influenza antibodies require Fc receptor engagement for in vivo protection. J. Clin. Invest. 126, 605-610 (2016). 


\section{Article}

6. DiLillo, D. J., Tan, G. S., Palese, P. \& Ravetch, J. V. Broadly neutralizing hemagglutinin stalk-specific antibodies require FcyR interactions for protection against influenza virus in vivo. Nat. Med. 20, 143-151 (2014)

7. Kallewaard, N. L. et al. Structure and function analysis of an antibody recognizing all influenza A subtypes. Cell 166, 596-608 (2016).

8. Corti, D. et al. A neutralizing antibody selected from plasma cells that binds to group 1 and group 2 influenza A hemagglutinins. Science 333, 850-856 (2011).

9. Bournazos, S. IgG Fc receptors: evolutionary considerations. Curr. Top. Microbiol. Immunol. 423, 1-11 (2019).

10. Smith, P., DiLillo, D. J., Bournazos, S., Li, F. \& Ravetch, J. V. Mouse model recapitulating human Fcy receptor structural and functional diversity. Proc. Natl Acad. Sci. USA 109 6181-6186 (2012).

11. Richards, J. O. et al. Optimization of antibody binding to FcyRlla enhances macrophage phagocytosis of tumor cells. Mol. Cancer Ther. 7, 2517-2527 (2008)

12. DiLillo, D. J. \& Ravetch, J. V. Differential Fc-receptor engagement drives an anti-tumor vaccinal effect. Cell 161, 1035-1045 (2015).

13. Weitzenfeld, P., Bournazos, S. \& Ravetch, J. V. Antibodies targeting sialyl Lewis A mediate umor clearance through distinct effector pathways. J. Clin. Invest. 129, 3952-3962 (2019).

14. Lazar, G. A. et al. Engineered antibody Fc variants with enhanced effector function. Proc. Natl Acad. Sci. USA 103, 4005-4010 (2006)

15. Ferrara, C. et al. Unique carbohydrate-carbohydrate interactions are required for high affinity binding between FcyRIII and antibodies lacking core fucose. Proc. Natl Acad. Sci. USA 108, 12669-12674 (2011).

16. Mimoto, F. et al. Engineered antibody Fc variant with selectively enhanced FcyRllb binding over both FcyRlla(R131) and FcyRlla(H131). Protein Eng. Des. Sel. 26, 589-598 (2013).

17. Wrammert, J. et al. Broadly cross-reactive antibodies dominate the human B cell response against 2009 pandemic H1N1 influenza virus infection. J. Exp. Med. 208, 181-193 (2011)
18. Eisenbarth, S. C. Dendritic cell subsets in T cell programming: location dictates function. Nat. Rev. Immunol. 19, 89-103 (2019).

19. Badia, R. et al. CD32 expression is associated to T-cell activation and is not a marker of the HIV-1 reservoir. Nat. Commun. 9, 2739 (2018).

20. Martin, G. E. et al. CD32-expressing CD4 T cells are phenotypically diverse and can contain proviral HIV DNA. Front. Immunol. 9, 928 (2018)

21. Descours, B. et al. CD32a is a marker of a CD4 T-cell HIV reservoir harbouring replication-competent proviruses. Nature 543, 564-567 (2017)

22. Wang, T. T. et al. IgG antibodies to dengue enhanced for FcyRIIIA binding determine disease severity. Science 355, 395-398 (2017).

23. Monsalvo, A. C. et al. Severe pandemic $2009 \mathrm{H} 1 \mathrm{~N} 1$ influenza disease due to pathogenic immune complexes. Nat. Med. 17, 195-199 (2011).

24. Mehta, P. et al. COVID-19: consider cytokine storm syndromes and immunosuppression. Lancet 395, 1033-1034 (2020).

25. Zalevsky, J. et al. Enhanced antibody half-life improves in vivo activity. Nat. Biotechnol. 28, 157-159 (2010).

26. Goede, V. et al. Obinutuzumab plus chlorambucil in patients with CLL and coexisting conditions. N. Engl. J. Med. 370, 1101-1110 (2014).

27. Nishimura, Y. et al. Early antibody therapy can induce long-lasting immunity to SHIV. Nature 543, 559-563 (2017)

28. Niessl, J. et al. Combination anti-HIV-1 antibody therapy is associated with increased virus-specific T cell immunity. Nat. Med. 26, 222-227 (2020).

Publisher's note Springer Nature remains neutral with regard to jurisdictional claims in published maps and institutional affiliations.

(c) The Author(s), under exclusive licence to Springer Nature Limited 2020 


\section{Methods}

\section{Viruses, cell lines and mouse strains}

The A/Puerto Rico/8/34 (PR8) and A/Netherlands/602/09 (Neth09) H1N1 viruses were grown as previously described ${ }^{6}$. MDCK cells (ATCC) were maintained at $37^{\circ} \mathrm{C}, 5 \% \mathrm{CO}_{2}$ in DMEM supplemented with $10 \% \mathrm{FBS}, 50 \mathrm{U} \mathrm{ml}^{-1}$ penicillin and $50 \mathrm{\mu g} \mathrm{ml}^{-1}$ streptomycin (ThermoFisher). Expi293F cells (ThermoFisher) were maintained at $37^{\circ} \mathrm{C}$, $8 \% \mathrm{CO}_{2}$ in Expi293 expression medium (ThermoFisher) supplemented with $10 \mathrm{U} \mathrm{ml}^{-1}$ penicillin and $10 \mu \mathrm{g} \mathrm{ml}^{-1}$ streptomycin. Fc $\gamma$ RIIa- and Fc $\gamma$ RIIIa-expressing NFAT reporter cells were purchased from Promega and have been authenticated by the vendor by STR analysis. All cell lines tested negative for mycoplasma contamination. All in vivo experiments were performed in compliance with federal laws and institutional guidelines and have been approved by the Rockefeller University Institutional Animal Care and Use Committee. Mice were bred and maintained at the Comparative Bioscience Center at the Rockefeller University at a controlled ambient temperature environment with 12-h dark/light cycle. C57BL/6 mice were purchased from The Jackson Laboratory. Fc $\mathrm{R}$-humanized mice $\left(\mathrm{mFc} \gamma \mathrm{R}^{-/-}, \mathrm{Fcgr1}^{-/-}\right.$,

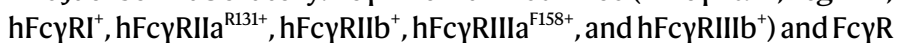
knockout mice $\left(\mathrm{mFc}_{\mathrm{R} \alpha^{-/-}} ; \mathrm{Fcgr1}^{-/-}\right)$were generated in the $\mathrm{C} 57 \mathrm{BL} / 6$ background and extensively characterized in previous studies ${ }^{10}$. Human Fc $\gamma$ RIIa-only mice $\left(\mathrm{hFc} \gamma \mathrm{RIIa}^{+}\right)$were generated in a mouse $\mathrm{Fc} \gamma \mathrm{R}$-deficient background (C57BL/6; Fc $\left.\gamma \mathrm{R \alpha}^{-/-} ; F c g r 1^{--}\right)^{10}$. FcRn-humanized mice (B6. Cg-Fcgrt $\left.{ }^{\mathrm{tmIDcr}} \mathrm{Tg}(F C G R T) 32 \mathrm{Dcr} / \mathrm{Dcr}\right)$ were purchased from The Jackson Laboratory and are deficient in mouse FcRn and express human FcRn as a transgene ${ }^{29,30}$. Fc $\gamma \mathrm{R} / \mathrm{FcRn}$-humanized mice were generated by crossing the Fc $\gamma$ R-humanized strain to the FcRn-humanized mice ${ }^{31}$.

\section{Cloning, expression and purification of recombinant IgG antibodies}

For the generation of Fc domain variants of human IgG1 Fc domain variants, site-directed mutagenesis using specific primers was performed as previously described ${ }^{4}$. Recombinant antibodies were generated by transient transfection of Expi293 or Expi-CHO cells with heavy and light chain expression plasmids using previously described proto$\operatorname{cols}^{13}$. Before transfection, plasmid sequences were validated by direct sequencing (Genewiz). Recombinant IgG antibodies were purified from cell-free supernatants by affinity purification using protein $\mathrm{G}$ or protein A sepharose beads (GE Healthcare). Purified proteins were dialysed in PBS, filter-sterilized $(0.22 \mu \mathrm{m})$, and purity was assessed by SDS-PAGE followed by SafeStain blue staining (ThermoFisher). All antibody preparations were more than $90 \%$ pure and endotoxin levels were less than $0.05 \mathrm{EU} \mathrm{mg}^{-1}$, as measured by the limulus amebocyte lysate assay. For the generation of afucosylated Fc domain variants, $\mathrm{CHO}$ cells were transfected with heavy chain and light chain expression plasmids in the presence of $100 \mu \mathrm{M} 2$-fluorofucose peracetate ${ }^{32}$. To confirm the absence of fucose, glycans were released with PNGase F, labelled with Waters RapiFluor-MS, cleaned up with a HILIC microElution plate, injected onto a Waters Glycan BEH Amide column, using a Thermo Vanquish UHPLC with FLD detection. Chromatograms were integrated and the relative contribution of each glycan calculated as a percentage. Peaks were identified by mass spec using a Thermo $Q$ Exactive Plus mass spectrometer and through comparison to the NIST monoclonal antibody standard. Size exclusion chromatography (SEC) was performed using a Superdex 200 Increase 10/300GL column (GE Healthcare) on an Äkta Pure 25 HPLC system and analysed using Unicorn v.6.3 software.

\section{ELISA-based Fc $\gamma \mathrm{R}$ binding assay}

Recombinant human Fc $\gamma$ R ectodomains (Sinobiological) $\left(5 \mu \mathrm{g} \mathrm{ml}^{-1}\right)$ were immobilized into high-binding 96-well microtitre plates (Nunc), and after overnight incubation at $4{ }^{\circ} \mathrm{C}$, plates were blocked with PBS plus $2 \%(\mathrm{w} / \mathrm{v}) \mathrm{BSA}$ and $0.05 \%(\mathrm{v} / \mathrm{v})$ Tween 20 for $2 \mathrm{~h}$. IgG immune complexes were prepared by incubating for $1 \mathrm{~h}$ at $4^{\circ} \mathrm{C}$ Fc variants of the anti-NP (4-hydroxy-3-nitrophenylacetyl) monoclonal antibody $\mathrm{Ab}$ 3B62 with NP-BSA (27 conjugation ratio, Biosearch Technologies) at a molar ratio of 1:10 (antigen:antibody). IgG immune complexes or monomeric IgG (for $\mathrm{Fc} \gamma \mathrm{RI}$ ) were serially diluted (1:3 consecutive dilutions in PBS starting at $10 \mu \mathrm{g} \mathrm{ml}^{-1}$ (IgG concentration) for immune complexes or $1 \mu \mathrm{g} \mathrm{ml}^{-1}$ for monomeric IgG) and applied to Fc $\gamma \mathrm{R}$-coated plates. After $1 \mathrm{~h}$ incubation at room temperature, bound IgG was detected using horseradish peroxidase (HRP)-conjugated goat $\mathrm{F}\left(\mathrm{ab}^{\prime}\right)_{2}$ anti-human IgG (1 h; 1:5,000;Jackson Immunoresearch). Plates were developed using the $\operatorname{TMB}\left(3,3^{\prime}, 5,5^{\prime}\right.$-tetramethylbenzidine $)$ two-component peroxidase substrate kit (KPL) and reactions were stopped with the addition of $1 \mathrm{M}$ phosphoric acid. Absorbance at $450 \mathrm{~nm}$ was immediately recorded using a SpectraMax Plus spectrophotometer (Molecular Devices) and background absorbance from negative control samples was subtracted. Data were collected and analysed using SoftMax Pro v.7.0.2 software (Molecular Devices).

\section{Surface plasmon resonance}

All experiments were performed with a Biacore T200 SPR system (GE Healthcare) at $25^{\circ} \mathrm{C}$ in HBS-EP ${ }^{+}$buffer (10 mM HEPES, pH 7.4, 150 $\mathrm{mM} \mathrm{NaCl}, 3.4$ mM EDTA, 0.005\% (v/v) surfactant P20). IgG antibodies (clone 3B62) were immobilized on Series S Protein G sensor chip (GE Healthcare) at a density of 2,000 response units (RU). Serial dilutions of recombinant soluble human Fc $\gamma$ RI ectodomain (Sinobiological) were injected to the flow cells at $20 \mu \mathrm{min}^{-1}$, with the concentration ranging from 250 to $0.488 \mathrm{nM}$ (1:2 successive dilutions). Association time was 60 s followed by a 900 -s dissociation step. At the end of each cycle, sensor surface was regenerated with a glycine $\mathrm{HCl}$ buffer $(10 \mathrm{mM}$, $\mathrm{pH} 2.0 ; 50 \mu \mathrm{l} \mathrm{min}^{-1}, 30 \mathrm{~s}$ ). Background binding to blank immobilized flow cells was subtracted, and affinity constants were calculated using BIAcore T200 evaluation software v.2.0 (GE Healthcare) using the 1:1 Langmuir binding model.

\section{Determination of antibody-dependent activation of human FcүRIIIa}

Monoclonal antibodies were serially diluted in ADCC assay buffer (Promega). Target cells (A549-H1 HA, A/California/04/2009) were added in a white flat-bottom 96-well plate at $1.25 \times 10^{4}$ cells per well in $25 \mu \mathrm{l}$, then serially diluted antibodies were added to each well ( $25 \mu \mathrm{l}$ per well), and the antibody and cell mixture was incubated for $10 \mathrm{~min}$ at room temperature. Effector cells (Jurkat-FcyRIIIa) for the ADCC Bioassay are thawed and added at a cell density of $7.5 \times 10^{4}$ per well in $25 \mu \mathrm{l}$ (effector to target ratio of $6: 1$ ). Plates were incubated for $20 \mathrm{~h}$ at $37^{\circ} \mathrm{C}$ with $5 \%$ $\mathrm{CO}_{2}$. Activation of human Fc $\gamma \mathrm{RIII}$ (V158 or F158 variants) in this bioassay results in the NFAT-mediated expression of the luciferase reporter gene. Luminescence is therefore measured with a luminometer (Synergy H1, Biotek) using the BioGlo Luciferase Assay Reagent according to the manufacturer's instructions. The data (that is, specific Fc $\gamma$ RIIIa activation) are expressed as the average of relative luminescence units (RLU) over the background by applying the following formula: (RLU at concentration $x$ of monoclonal antibodies - RLU of background).

\section{Determination of antibody-dependent activation of human FcyRIIa}

Monoclonal antibodies were serially diluted in ADCP assay buffer (Promega). Target cells (A549-H1HA, A/California/04/2009) were added in a white flat-bottom 96-well plate at $10^{4}$ cells per well in $25 \mu \mathrm{l}$, then serially diluted antibodies were added to each well ( $25 \mu \mathrm{l}$ per well), and the antibody and cell mixture was incubated for $10 \mathrm{~min}$ at room temperature. Effector cells (Jurkat-Fc $\gamma$ RIIa) for the ADCP Bioassay are thawed and added at a cell density of $5 \times 10^{4}$ per well in $25 \mu \mathrm{l}$ (effector to target ratio of 5:1). Plates were incubated for $20 \mathrm{~h}$ at $37^{\circ} \mathrm{C}$ with $5 \% \mathrm{CO}_{2}$. Activation of human Fc $\gamma$ RIIa (H131 variant) in this bioassay results in the NFAT-mediated expression of the luciferase reporter 
gene. Luminescence is therefore measured with a luminometer (Synergy H1, Biotek) using the BioGlo Luciferase Assay Reagent according to the manufacturer's instructions. The data (that is, specific Fc $\gamma$ RIla activation) are expressed as the average of relative luminescence units (RLU) over the background by applying the following formula: (RLU at concentration $x$ of antibodies - RLU of background).

\section{ADCC assay}

Natural killer cells were freshly isolated from whole EDTA blood using the MACSxpress NK isolation kit following the manufacturer instruction. Monoclonal antibodies were serially diluted tenfold in AIM-V medium from $1 \mu \mathrm{g} \mathrm{ml}^{-1}$ to $0.001 \mu \mathrm{g} \mathrm{ml}^{-1}$. Target cells (A549-H1HA, $\mathrm{A} /$ California/04/2009) were added in a round-bottom 384-well plate at $7.5 \times 10^{3}$ cells per well in $23 \mu \mathrm{l}$, then serially diluted antibodies were added to each well ( $23 \mu \mathrm{l}$ per well), and the antibody-cell mixture was incubated for $10 \mathrm{~min}$ at room temperature. After incubation, human natural killer cells were added at a cell density of $4.5 \times 10^{4}$ per well in $23 \mu \mathrm{l}$ (effector to target ratio of 6:1). Control wells were also included that were used to measure maximal lysis (containing target cells with $23 \mu \mathrm{l}$ of $3 \%$ Triton X-100) and spontaneous lysis (containing target cells and effector cells without antibody). Plates were incubated for $4 \mathrm{~h}$ at $37^{\circ} \mathrm{C}$ with $5 \% \mathrm{CO}_{2}$. Cell death was determined by measuring lactate dehydrogenase (LDH) release using an LDH detection kit (Roche) according to the manufacturer's instructions. Using a kinetic protocol, the absorbance at $490 \mathrm{~nm}$ and $650 \mathrm{~nm}$ was measured once every $2 \mathrm{~min}$ for $8 \mathrm{~min}$. The percentage of specific lysis was determined by applying the following formula: (specific release - spontaneous release)/(maximum release - spontaneous release) $\times 100$.

\section{Anti-HA, NA and NP ELISA}

Recombinant HA (Influenza A H1N1 (A/California/04/2009 or A/Puerto Rico/8/34) or H3N2 (A/x31)), or NA (A/California/04/2009) or NP(H1N1 (A/California/04/2009) or H3N2 (A/x31)) (Sinobiological) $\left(3 \mu \mathrm{g} \mathrm{ml}^{-1}\right)$ were immobilized into high-binding 96-well microtitre plates (Nunc) and after overnight incubation at $4{ }^{\circ} \mathrm{C}$, plates were blocked with PBS plus $2 \%(\mathrm{w} / \mathrm{v}) \mathrm{BSA}$ and $0.05 \%(\mathrm{v} / \mathrm{v})$ Tween 20 for $2 \mathrm{~h}$. After blocking, plates were incubated for $1 \mathrm{~h}$ with serially diluted IgG antibodies or serum samples ( $1: 3$ consecutive dilutions in PBS starting at $1 \mu \mathrm{g} \mathrm{ml}^{-1}$ for monoclonal antibodies or 1:10 for serum samples), followed by HRP-conjugated goat anti-human IgG (minimal cross-reactivity to mouse IgG) for human monoclonal antibodies or goat anti-mouse IgG (minimal cross-reactivity to human IgG) for serum samples ( $1 \mathrm{~h}$; 1:5,000; Jackson Immunoresearch). Plates were developed using the TMB two-component peroxidase substrate kit (KPL) and reactions were stopped with the addition of $1 \mathrm{M}$ phosphoric acid. Absorbance at 450 nm was immediately recorded using a SpectraMax Plus spectrophotometer (Molecular Devices) and background absorbance from negative control samples was subtracted. Data were collected and analysed using SoftMax Pro v.7.0.2 software (Molecular Devices).

\section{Microneutralization assay}

The neutralizing activity of anti-HA and NA monoclonal antibody Fc variants was evaluated in microneutralization assays, using previously described protocols ${ }^{33}$. Virus input was titrated to maximize the signal-to-noise ratio. Fc domain variants (starting concentration at $100 \mu \mathrm{g} \mathrm{ml}^{-1}$ followed by $1: 3$ serial dilutions) and viruses $\left(1.8 \times 10^{3} \mathrm{pfu} \mathrm{ml}^{-1}\right.$ for A/Puerto Rico/8/34 and $3.2 \times 10^{4} \mathrm{pfu} \mathrm{ml}^{-1}$ for A/Netherlands/602/09) were prepared in DMEM supplemented with $50 \mathrm{U} \mathrm{ml}^{-1}$ penicillin,

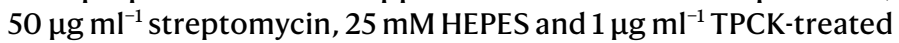
trypsin (Sigma). The virus-antibody mixture was pre-incubated for $1 \mathrm{~h}$ at $37^{\circ} \mathrm{C}$ and added to a monolayer of MDCK cells (70-80\% confluent in 96-well plates). After incubation at $37^{\circ} \mathrm{C}$ for $1 \mathrm{~h}$ to allow for virus adsorption, the cell monolayer was washed three times with PBS and re-incubated for $18-20 \mathrm{~h}$ at $37^{\circ} \mathrm{C}$ with medium (DMEM supplemented with $50 \mathrm{U} \mathrm{ml}^{-1}$ penicillin, $50 \mathrm{\mu g} \mathrm{ml}^{-1}$ streptomycin, $25 \mathrm{mM}$ HEPES and
$1 \mu \mathrm{g} \mathrm{ml}^{-1}$ TPCK-treated trypsin) containing monoclonal antibodies (at equivalent concentrations as during the virus co-incubation). Cells were fixed with $80 \%(\mathrm{v} / \mathrm{v})$ acetone, blocked with $5 \%(\mathrm{w} / \mathrm{v})$ non-fat milk diluted in PBS for 30 min at room temperature, and quenched with $3 \%(v / v)$ hydrogen peroxide (in PBS) by incubating for a further $20 \mathrm{~min}$ at room temperature. Cells were stained with biotinylated anti-NP antibody (EMD Millipore; 1:2,000), followed by HRP-conjugated streptavidin (Jackson Immunoresearch;1:5,000). Plates were developed using the TMB two-component peroxidase substrate kit (KPL) and reactions stopped with the addition of $1 \mathrm{M}$ phosphoric acid. Absorbance at 450 nm was immediately recorded using a SpectraMax Plus spectrophotometer (Molecular Devices) and background absorbance from negative control samples was subtracted. Data were collected and analysed using SoftMax Pro v.7.0.2 software (Molecular Devices).

\section{Haemagglutination inhibition assay}

$\mathrm{HAI}$ activity was evaluated using previously described protocols ${ }^{34}$. In brief, serum samples (diluted 1:3 in cholera filtrate) or Fc domain variants of antibodies (starting concentration at $100 \mu \mathrm{g} \mathrm{ml}^{-1}$ followed by $1: 3$ serial dilutions) and viruses (A/Puerto Rico/8/34 or $\mathrm{A} /$ Netherlands/602/09; $10^{7} \mathrm{pfu} \mathrm{ml}^{-1}$ ) were incubated in V-bottom 96 microtitre plates for $30 \mathrm{~min}$ at room temperature. Turkey red blood cells $(0.75 \%$ (v/v); Rockland) were added to the antibody-virus mixture, mixed gently, and incubated for $30 \mathrm{~min}$ at room temperature. Plates were scored for the number of wells exhibiting HAI activity.

\section{Quantification of serum IgG levels}

Blood from mice was collected into gel microvette tubes, serum was fractionated by centrifugation $(10,000 \mathrm{~g}, 5 \mathrm{~min})$ and stored at $-20^{\circ} \mathrm{C}$. IgG levels in serum samples were determined by ELISA following previously published protocols ${ }^{13,31}$.

\section{Mouse influenza infection models}

No statistical method was used to predetermine sample size. On the basis of preliminary studies that determined experimental variation in survival after infection and antibody treatment, we performed power calculations and determined that at least $n=6$ mice per group is sufficient to detect differences among experimental groups (powered at $80 \%$ for $5 \%$ significance level; survival assessed by log-rank(Mantel-Cox) test). Mice(females; 6-12 weeks old) were anaesthetized with a ketamine $\left(75 \mathrm{mg} \mathrm{kg}^{-1}\right)$ and xylazine $\left(15 \mathrm{~g} \mathrm{~kg} \mathrm{k}^{-1}\right.$ ) mixture (administered inraperitoneally) and viruses (diluted in PBS) were administered intranasally (five $\mathrm{mLD}_{50}$ ) in $30 \mu$ l. After infection, mice were monitored daily, and their weights were recorded for 14 days. Death was determined by a $20 \%$ body weight loss threshold that was authorized by the Rockefeller University Institutional Animal Care and Use Committee. Mice were randomized based on age and weight. Before treatment, we ensured that the mean weight and age were comparable among the various treatment groups. Whenever possible (notalways, due tolimited access of laboratory staff in BSL-2 animal facilities), the treatment groups were blinded to the person involved in monitoring mouse survival and weight upon challenge (applicable to data presented in Figs. 1d, 4). Forantibody-mediated prophylaxis, antibodieswereadministered intraperitoneally or intravenously $4 \mathrm{hbefore}$ irus challenge (except for experiments with FcRn/FcyR-humanized mice, in which antibodies were administered 2 daysbefore infection), whereas for antibody-mediated therapy, antibodies were administered on day 3 after infection. Antibody dose was calculated as $\mathrm{mg} \mathrm{kg}^{-1}$. For Fc $\gamma \mathrm{RIla}$-blocking experiments, recombinant anti-Fc $\gamma \mathrm{RIIa}$ (clone IV.3) expressed as human IgG1 GRLR variant to abrogate Fc $\gamma$ R binding or isotype control (anti-hapten (NP) monoclonal antibody; clone 3C13) was administered $(80 \mu \mathrm{g})$ intranasally to mice 1 day after virus challenge.

\section{Ex vivo stimulation of human monocyte-derived dendritic cells} Leukocyte packs (buffy coats) were purchased from the New York Blood Center, mononuclear cells were isolated by Ficoll gradient centrifugation, and $\mathrm{CD}_{14}{ }^{+}$monocytes were purified using CD14 
microbeads (Miltenyi Biotech). Cells were cultured at $37^{\circ} \mathrm{C}, 5 \% \mathrm{CO}_{2}$ for 6 days in RPMI supplemented with $50 \mathrm{U} \mathrm{ml}^{-1}$ penicillin, $50 \mathrm{\mu g} \mathrm{ml}^{-1}$ streptomycin, $10 \%$ heat-inactivated FBS, $100 \mathrm{ng} \mathrm{ml}^{-1} \mathrm{GM}-\mathrm{CSF}$, and $100 \mathrm{ng} \mathrm{ml}^{-1} \mathrm{IL}-4$. Cells were then seeded in 96 -well plates at $8 \times 10^{4}$ cells per well in RPMI supplemented with $50 \mathrm{U} \mathrm{ml}^{-1}$ penicillin, $50 \mathrm{\mu g} \mathrm{ml}^{-1}$ streptomycin, 5\% ultra-low IgG, heat-inactivated FBS and stimulated overnight with IgG immune complexes $\left(100 \mu \mathrm{g} \mathrm{ml}^{-1}\right)$. The following immune complexes were used: (i) heat-aggregated IgG complexes generated by incubation of IgG (1A01 monoclonal antibody) at $63^{\circ} \mathrm{C}$ for 60 min; or (ii) anti-NP:NP-BSA immune complexes generated as described for the ELISA-based Fc $\gamma$ R binding assay. After overnight stimulation, dendritic cell maturation was assessed by flow cytometry. Cells were stained with the following fluorochrome-conjugated antibodies (diluted at 1:100 unless otherwise stated): anti-DC-SIGN-BrilliantViolet 421, anti-CD40-BrilliantViolet 510, anti-human FcyRI-BrilliantViolet 605 (clone 10.1), anti-CD11b-BrilliantViolet 650, anti-CD86-BrilliantViolet 711, anti-human FcyRIIa-FITC (clone IV.3, used at 1:50 dilution), anti-CD11c-PerCP-Cy5.5, anti-human FcyRIIIa/b-PE (clone 3G8), anti-CD80-PE/Cy7, anti-human Fc $\gamma$ RIlb-Dylight 650 (clone 2B6) (used at $10 \mathrm{gg} \mathrm{ml}^{-1}$ ), anti-HLA-DR-AlexaFluor700, and anti-CD83-APC/Cy7.Samples were collected on an Attune NxT flow cytometer (ThermoFisher) using Attune NxT software v.3.1.2 and analysed using FlowJo (v.10.6) software.

\section{In vivo neutrophil, $\mathrm{CD8}^{+}$or $\mathrm{CD}^{+} \mathrm{T}$ cell depletion}

Neutrophils, $\mathrm{CD}^{+}$or $\mathrm{CD}^{+}$cells were depleted in mice by administration of anti-Gr-1, anti-CD8 or anti-CD4 monoclonal antibodies, respectively. To establish the efficiency of antibody-mediated cell depletion, Fc $\gamma$ R-humanized mice were injected intravenously with 150 $\mu \mathrm{g}$ anti-mouse $\mathrm{Gr}-1$ monoclonal antibody (clone RB6-8C5; rat IgG2b; Bioxcell), anti-mouse CD8 $\alpha$ monoclonal antibody (clone 2.43; rat IgG2b; Bioxcell), anti-mouse CD4 monoclonal antibody (clone GK1.5; rat IgG2b; Bioxcell), or isotype control (clone LTF-2; rat IgG2b; Bioxcell). The abundance of neutrophils, $\mathrm{CD}^{+}$and $\mathrm{CD}^{+}{ }^{+}$T cells in peripheral blood was determined at various time points after antibody administration by flow cytometry. Baseline $\mathrm{CD}^{+}$and $\mathrm{CD} 4^{+} \mathrm{T}$ cell frequencies were determined in blood samples obtained before antibody administration. For the flow cytometry analysis, fluorescently conjugated monoclonal antibodies targeting Ly6G (clone 1A8; Biolegend), the $\beta$ subunit of mouse CD8 (clone YTS156.7.7; Biolegend) or epitopes non-overlapping with the GK1.5 epitope (clone RM4-4; Thermofisher) were used to avoid competition with the depleting antibodies. Neutrophil, $\mathrm{CD}^{+}$or $\mathrm{CD}^{+}$ $T$ cell depletion of influenza-infected mice was performed using the aforementioned conditions and depleting antibodies or isotype were administered intravenously $(150 \mu \mathrm{g}$ ) on day 1 (for neutrophils) or 3 (for $\mathrm{CD}^{+}$or $\mathrm{CD}^{+} \mathrm{T}$ cells) after infection.

\section{Processing of mouse tissues and flow cytometry analysis}

Mice were euthanized and lungs were perfused by injection of PBS (containing $10 \mathrm{U} \mathrm{ml}^{-1}$ heparin) into the right cardiac ventricle. Lungs were excised and homogenized using the gentleMACS dissociator (mouse lung dissociation kit, Miltenyi), according to the manufacturer's recommendations. Spleens were homogenized by mechanical shearing. After lysis of red blood cells (RBC lysis buffer; Biolegend), single-cell suspensions were labelled with the LIVE/DEAD Fixable Near-IR (ThermoFisher) and resuspended in PBS containing 0.5\% $(\mathrm{w} / \mathrm{v}) \mathrm{BSA}$ and $5 \mathrm{mM}$ EDTA. Cells were labelled with mixtures of fluorescently labelled antibodies including (all used at 1:250 dilution unless otherwise stated): (i) for the characterization of the Fc $\gamma$ RIIa expression of the Fc $\mathrm{RIIa}^{+}$mouse strain: anti-CD11c-eFluor506, anti-CD11b-BrilliantViolet605, anti-SiglecF-SuperBright645, anti-CD8 $\beta$-BrilliantViolet711, anti-MHCII- BrilliantViolet785, anti-human Fc $\gamma$ RIIa (clone IV.3)-FITC (used at 1:50 dilution), anti-Gr1-PerCP/Cy5.5, anti-CD3-PE, anti-CD103-PE/eFluor610, anti-NK1.1-PE/ Cy7, anti-CD4-AlexaFluor647, and anti-CD19-AlexaFluor700; (ii) for the evaluation of $\mathrm{Fc} \gamma \mathrm{R}$ expression on innate effector leukocytes:
anti-CD11c-eFluor506, anti-human FcүRI (clone 10.1)-BrilliantViolet605 (used at 1:100 dilution), anti-SiglecF-SuperBright645, anti-Ly6GBrilliantViolet711, anti-CD11b-BrilliantViolet785, anti-human FcyRIIa (clone IV.3)-FITC (used at 1:50 dilution), anti-Ly6C-PerCP/Cy5.5, anti-human FcyRIIIa/b (clone 3G8)-PE (used at 1:100 dilution), anti-CD103-PE/eFluor610, anti-NK1.1-PE/Cy7, and anti-human Fc $\gamma$ RIIb (clone 2B6)-Dylight650 (used at $10 \mu \mathrm{g} / \mathrm{ml}$ ); (iii) for the evaluation of Fc $\gamma$ R expression and activation status of DCs: anti-CD11c-eFluor506, anti-human FcyRI (clone 10.1)-BrilliantViolet605 (used at 1:100 dilution), anti-SiglecF-SuperBright645, anti-CD80-BrilliantViolet711, anti-CD11b-BrilliantViolet785, anti-human Fc $\gamma$ RIIa (clone IV.3)-FITC (used at 1:50 dilution), anti-Gr-1-PerCP/Cy5.5, anti-human FcyRIIIa/b (clone 3G8)-PE (used at 1:100 dilution), anti-CD103-PE/eFluor610, anti-CD86-PE/Cy7, anti-human Fc $\gamma$ RIIb (clone 2B6)-Dylight650 (used at $10 \mu \mathrm{g} \mathrm{ml}^{-1}$ ), and anti-MHCII-AlexaFluor700; (iv) for the evaluation of CD8 or CD4 depletion: anti-CD3e-eFluor506, anti-CD19-BrilliantViolet605, anti-CD8 $\beta$-BrilliantViolet711, anti-CD11b-PE, anti-NK1.1-PE/Cy7, antiCD4-FITC, anti-Gr-1-PerCP/Cy5.5, anti-NKp46-eFluor660, and anti-B220APC/eFluor780; ( $v$ ) for the evaluation of neutrophil depletion: antiCD11c-eFluor506, anti-CD19-BrilliantViolet605, anti-SiglecF-SuperBright645, anti-Ly6G-BrilliantViolet711, anti-CD11b-BrilliantViolet785, anti-CD8a-FITC, anti-Ly6C-PerCP/Cy5.5, anti-CD3-PE, anti-NK1.1-PE/ Cy7, anti-CD4-AlexaFluor647, anti-CD45-AlexaFluor700; (vi) for the assessment of Fc $\gamma$ R expression on T cells: anti-B220-BrilliantViolet421, anti-CD3-BrilliantViolet510, anti-human FcyRI (clone 10.1)BrilliantViolet605 (used at 1:100 dilution), anti-CD8-BrilliantViolet711, anti-CD4-BrilliantViolet785, anti-human FcyRIIa (clone IV.3)-FITC (used at 1:50 dilution), anti-NK1.1-PerCP/Cy5.5, anti-human Fc $/$ RIIIa/b (clone 3G8)-PE (used at 1:100 dilution), anti-CD11b-PE/Cy7, anti-human FcyRIlb (clone 2B6)-Dylight650 (used at $10 \mu \mathrm{g} \mathrm{ml}^{-1}$ ), and anti-Gr-1-AlexaFluor 700; (vii) for the characterization of dendritic cell populations after antibody treatment: anti-CD103-FITC, anti-Ly6C-PerCP/Cy5.5, antiNK1.1-AlexaFluor647, anti-CD45-AlexaFluor700, anti-CD11c-eFluor506, anti-CD86-BrilliantViolet605, anti-SiglecF-SuperBright645, antiLy6G-BrilliantViolet711, anti-CD11b-BrilliantViolet785, anti-CD40PE, anti-MHCII-PE/eFluor610, and anti-CD80-PE/Cy7; (viii) for the characterization of $\mathrm{T}$ cell populations after antibody treatment: antiCD4-AlexaFluor488, anti-CD3e-PerCP/Cy5.5, anti-NK1.1-AlexaFluor647, anti-CD45-AlexaFluor700, anti-CD44-BrilliantViolet421, antiCD62L-BrilliantViolet510, anti-CD25-BrilliantViolet605, antiCD27-BrilliantViolet650, anti-CD8-BrilliantViolet711, anti-CD11a-PE, anti-CCR7-PE/eFluor610, and anti-CD69-PE/Cy7. For experiments assessing $\mathrm{Fc} \gamma \mathrm{R}$ expression, relevant isotype control antibodies were used and included: mouse IgG1 isotype control-Dylight650 (used at $10 \mu \mathrm{g} \mathrm{ml}^{-1}$ ), mouse IgG2b kappa isotype control-FITC (used at 1:50 dilution), mouse IgG1 kappa isotype control-PE (used at 1:100 dilution), mouse IgG1 kappa isotype control-BrilliantViolet605 (used at 1:100 dilution). Cell counts were determined using CountBright absolute counting beads (ThermoFisher). Samples were collected on an Attune NxT flow cytometer (ThermoFisher) using Attune NxT software v3.1.2 and analysed using FlowJo (v10.6) software. For cluster analysis, dendritic cells (defined as Live/ $\mathrm{Lin}^{+} / \mathrm{CD} 45^{+} / \mathrm{CD}_{11 \mathrm{c}^{+}} / \mathrm{MHCII}^{+}$) and T cells (defined as Live/CD $45^{+} / \mathrm{NK} .1^{\prime} / \mathrm{CD}^{+}$) from individual mice were downsampled using the Downsample v.3.2 plugin (3,000 (dendritic cells) or 6,000 (T cells) events/mouse; 12,000 (dendritic cells) or 24,000 (T cells)/ treatment condition) and concatenated. Cells were clustered and visualized using UMAP reduction (UMAP plugin v2.2) and populations were identified by KNN density estimation (X-shift, v.1.3) ${ }^{35}$.

\section{Analysis of Fc $\gamma \mathrm{R}$ expression on human T cells}

Leukocyte packs (buffy coats) were purchased from the New York Blood Center, mononuclear cells were isolated by Ficoll gradient centrifugation and stained with the following fluorochrome-conjugated anti-human antibodies: anti-CD14-BrilliantViolet510, anti-CD19-BrilliantViolet510, anti-human FcyRI-BrilliantViolet605 


\section{Article}

(clone 10.1), anti-CD8-BrilliantViolet650, anti-CD3-BrilliantViolet711, anti-CD11b-BrilliantViolet785, anti-human FcyRIla-FITC (clone IV.3) (used at 1:50 dilution), anti-CD4-PerCP-Cy5.5, anti-human FcyRIIIa/b-PE (clone 3G8), anti-CD56-PE/Cy7, anti-human Fc $\mathrm{RIIb}$-Dylight650 (clone 2B6) (used at $10 \mu \mathrm{g} \mathrm{ml}^{-1}$ ), anti-CD41-AlexaFluor700, and anti-CD42b-AlexaFluor700. The following isotype controls were used: mouse IgG1 isotype control-Dylight650 (used at $10 \mu \mathrm{g} \mathrm{ml}^{-1}$ ), mouse IgG2b kappa isotype control-FITC (used at 1:50 dilution), mouse IgG1 kappa isotype control-PE (used at 1:100 dilution), mouse IgG1 kappa isotype control-BrilliantViolet605 (used at 1:100 dilution). Samples were collected on an Attune NxT flow cytometer (ThermoFisher) using Attune NxT software v3.1.2 and analysed using FlowJo (v.10.6) software.

\section{Statistical analysis}

Results from multiple experiments are presented as mean \pm s.e.m. Oneor two-way ANOVA was used to test for differences in the mean values of quantitative variables, and where statistically significant effects were found, post hoc analysis using Bonferroni (adjusted for multiple comparisons) test was performed. Two-tailed $t$-test was used to test for differences in datasets with two groups. Statistical differences between survival rates were analysed by comparing Kaplan-Meier curves using the log-rank (Mantel-Cox) test. Data were analysed with GraphPadPrism v.8.4 software (GraphPad) and $P<0.05$ were considered to be statistically significant.

\section{Reporting summary}

Further information on research design is available in the Nature Research Reporting Summary linked to this paper.

\section{Data availability}

Source data are provided with this paper.

29. Petkova, S. B. et al. Enhanced half-life of genetically engineered human IgG1 antibodies in a humanized FcRn mouse model: potential application in humorally mediated autoimmune disease. Int. Immunol. 18, 1759-1769 (2006).
30. Roopenian, D. C., Christianson, G. J. \& Sproule, T. J. Human FcRn transgenic mice for pharmacokinetic evaluation of therapeutic antibodies. Methods Mol. Biol. 602, 93-104 (2010).

31. Borghi, S. et al. FcRn, but not FcyRs, drives maternal-fetal transplacental transport of human IgG antibodies. Proc. Natl Acad. Sci. USA 117, 12943-12951 (2020).

32. Okeley, N. M. et al. Development of orally active inhibitors of protein and cellular fucosylation. Proc. Natl Acad. Sci. USA 110, 5404-5409 (2013).

33. He, W., Mullarkey, C. E. \& Miller, M. S. Measuring the neutralization potency of influenza A virus hemagglutinin stalk/stem-binding antibodies in polyclonal preparations by microneutralization assay. Methods 90, 95-100 (2015).

34. Kaufmann, L. et al. An optimized hemagglutination inhibition (HI) assay to quantify influenza-specific antibody titers. J. Vis. Exp. 130, 55833 (2017).

35. Samusik, N., Good, Z., Spitzer, M. H., Davis, K. L. \& Nolan, G. P. Automated mapping of phenotype space with single-cell data. Nat. Methods 13, 493-496 (2016).

36. Dahan, R. et al. Therapeutic activity of agonistic, human anti-CD40 monoclonal antibodies requires selective FcyR engagement. Cancer Cell 29, 820-831 (2016).

Acknowledgements We thank R. Francis for technical assistance in antibody cloning and expression, in vitro experiments, flow cytometry, and in vivo influenza challenge studies, M. Ye and E. Lam for maintaining the mouse colonies and for assisting with in vitro and in vivo experiments, P. Smith for maintaining the humanized mouse strains and all the members of the Laboratory of the Molecular Genetics and Immunology for discussions. We also thank

S. Jaconi, K. Culap, E. Cameroni and B. Guarino for providing the FY1 monoclonal antibody Fc variants and for technical assistance in characterizing the in vitro $A D C C / A D C P$ activity of FC variants. Research reported in this publication was supported by the National Institute of Allergy and Infectious Diseases (R01Al129795 and R01Al145870). We acknowledge support from the Rockefeller University and Vir Biotechnology Inc. The content is solely the responsibility of the authors and does not necessarily represent the official views of the NIH.

Author contributions S.B. designed the study, performed experiments, analysed data, and wrote the manuscript; D.C. and H.W.V. provided intellectual input; J.V.R. designed the study and wrote the manuscript.

Competing interests S.B. and J.V.R. are inventors on a patent (WO2019125846A1) describing the GAALIE variant and its use in therapeutic monoclonal antibodies; D.C. and H.W.V. are employees of Vir Biotechnology Inc. and may hold shares in Vir Biotechnology Inc.; J.V.R. is a member of the scientific advisory board and a consultant of Vir Biotechnology Inc.

Additional information

Supplementary information is available for this paper at https://doi.org/10.1038/s41586-0202838-z.

Correspondence and requests for materials should be addressed to J.V.R.

Peer review information Nature thanks George Georgiou and the other, anonymous, reviewer(s) for their contribution to the peer review of this work. Peer reviewer reports are available.

Reprints and permissions information is available at http://www.nature.com/reprints. 

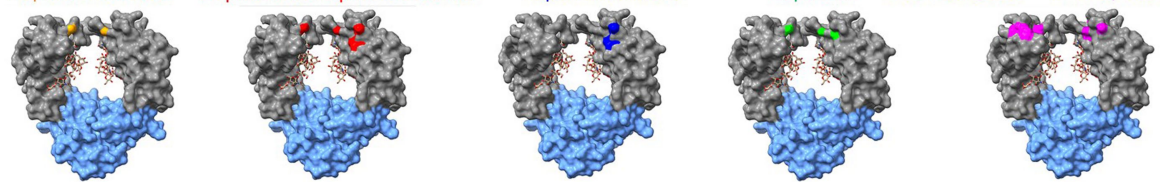

b

\begin{tabular}{|c|c|c|c|c|c|c|c|c|}
\hline & \multicolumn{2}{|c|}{ FcyRI } & \multicolumn{2}{|c|}{ FcyRIlaR131 } & \multicolumn{2}{|c|}{ FcyRllb } & \multicolumn{2}{|c|}{ FcyRIIIaF158 } \\
\hline & $\mathrm{KD}(\mathrm{M})$ & Fold & $\mathrm{KD}(\mathrm{M})$ & Fold & KD (M) & Fold & $\mathrm{KD}(\mathrm{M})$ & Fold \\
\hline Wild Type & $2.63 \times 10^{-10}$ & 1.00 & $* 2.91 \times 10^{-6}$ & 1.00 & $* 6.21 \times 10^{-6}$ & 1.00 & $* 1.43 \times 10^{-6}$ & 1.0 \\
\hline GA & $1.95 \times 10^{-9}$ & 0.13 & $* 4.88 \times 10^{-7}$ & 5.96 & $* 4.93 \times 10^{-6}$ & 1.26 & $* 1.12 \times 10^{-6}$ & 1.3 \\
\hline ALIE & $3.26 \times 10^{-11}$ & 8.07 & $* 3.51 \times 10^{-6}$ & 0.83 & $* 5.59 \times 10^{-6}$ & 1.11 & $* 1.17 \times 10^{-7}$ & 12.2 \\
\hline GAALIE & $3.16 \times 10^{-10}$ & 0.83 & ${ }^{*} 8.19 \times 10^{-7}$ & 3.55 & $* 1.96 \times 10^{-5}$ & 0.32 & $* 1.64 \times 10^{-7}$ & 8.7 \\
\hline V11 & $+2.30 \times 10^{-7}$ & 0.001 & $\dagger 3.84 \times 10^{-6}$ & 0.76 & $\dagger 3.15 \times 10^{-8}$ & 197 & tn.d.b. & \\
\hline GRLR & $<10^{-5}$ & & ‡n.d.b. & & ‡n.d.b. & & $\neq$ fn.d.b. & \\
\hline
\end{tabular}
KD values were determined by SPR using soluble human FcyR ectodomains. n.d.b.: no detectable binding; * KD reported in $13 ; \uparrow$ reported in $36 ; \neq$ reported in

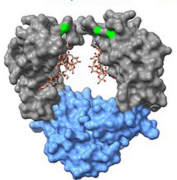

d

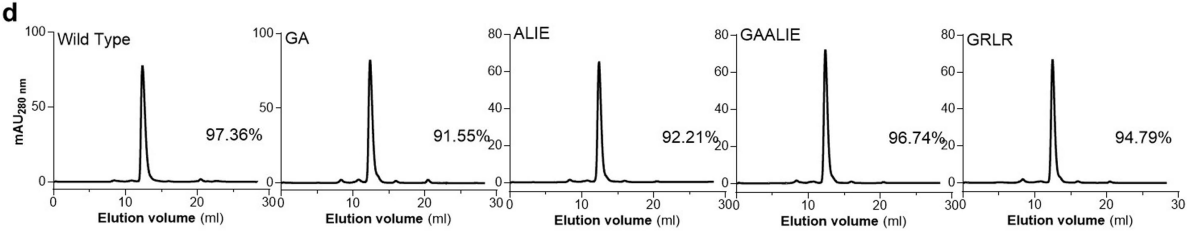

e
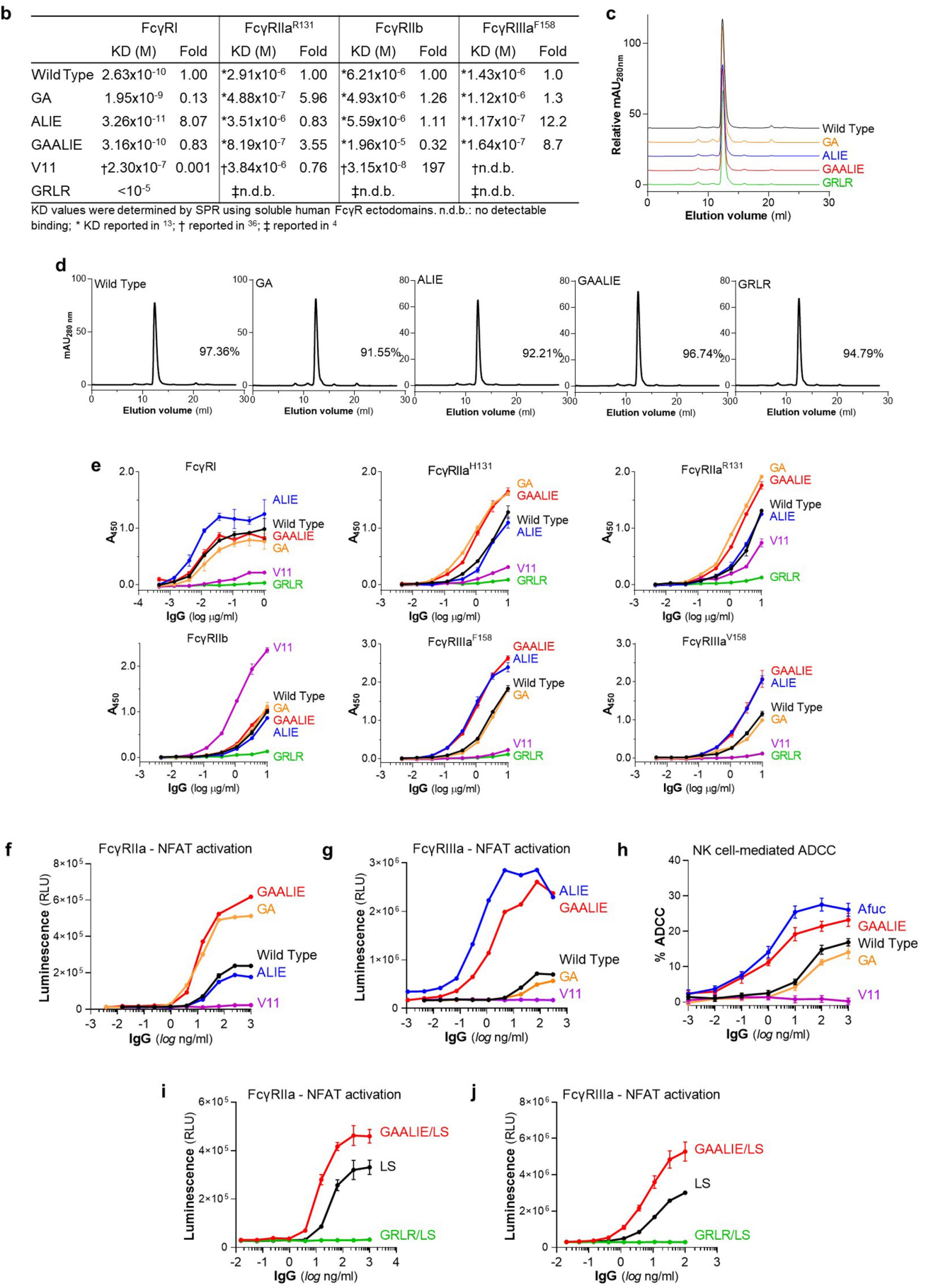

Extended Data Fig. 1 |See next page for caption. 


\section{Article}

Extended Data Fig. 1 Characterization of the Fc $\gamma \mathrm{R}$ binding profile and Fc effector activity of Fc domain variants. Fc domain variants with differential Fc $\gamma$ R binding affinity were generated through the introduction of amino acid substitutions at the hinge proximal region of the $\mathrm{CH} 2$ domain of human IgG1. a, The positions of the mutated residues for the different Fc domain variants are highlighted. b. The affinity of these human IgG1 Fc variants for the different human $\mathrm{Fc} \gamma \mathrm{Rs}$ was assessed by surface plasmon resonance and the dissociation constant $\left(K_{\mathrm{d}}\right)$ (in $\left.\mathrm{M}\right)$ is presented. The following references are cited in the table: refs. ${ }^{13,36}$.c, d, HPLC analysis of Fc domain variants using size-exclusion chromatography (SEC) columns was performed to determine whether mutations at the Fc domain are associated with increased antibody aggregation. The SEC profiles (c: overlay; d: individual Fc variants) and the abundance (percentage) of monomeric IgG is presented for the different Fc variants. e, Fc variants for the anti-NP monoclonal antibody $3 \mathrm{~B} 62$ were generated and their binding (monomeric for Fc $\gamma \mathrm{RI}$ or as IgG immune complexes using NP-BSA for all other Fc $\gamma \mathrm{Rs}$ ) to immobilized human Fc $\gamma \mathrm{RI}$, Fc $\gamma$ RIIa, Fc $\gamma$ RIIb and Fc $\gamma$ RIIIa was assessed by ELISA. Results are from one experiment performed in duplicates. The Fc effector activity of anti-influenza monoclonal antibody FY1 Fc variants was assessed in vitro using Fc $\gamma$ RIIaexpressing (f: $n=2$ independent experiments for wild type, $n=1$ for other groups, $\mathbf{i}: n=2$ independent experiments) and Fc $\gamma$ RIIIa-expressing ( $\mathbf{g}\left(\mathrm{F}^{158}\right.$ allele), $\mathbf{j}\left(\mathrm{V}^{158}\right.$ allele); $n=1$ for each variant, except for GAALIE-LS $(n=2$ independent experiments)) NFAT reporter cell lines. Fc variants with enhanced affinity for Fc $\gamma$ RIIa or Fc $\gamma$ RIIIa demonstrated increased capacity to induce NFAT reporter activation. $\mathbf{h}$, Similarly, FY1 Fc variants engineered for Fc $\gamma$ RIIIa binding exhibited improved primary human natural killer cell-mediated ADCC activity against HA-expressing cells. Results are the mean from two independent experiments using different natural killer cell donors. 
a

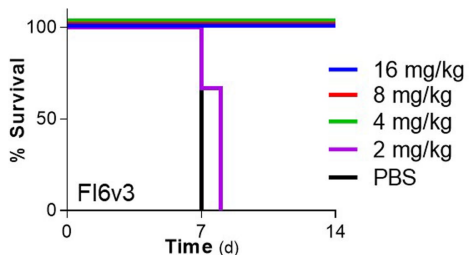

C

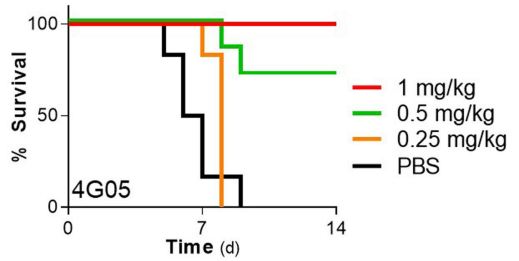

b

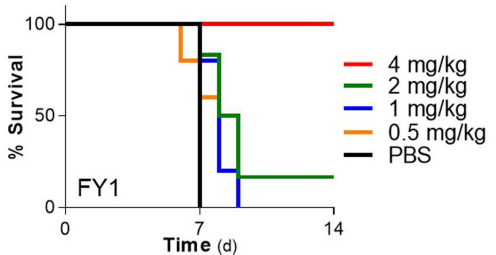

d

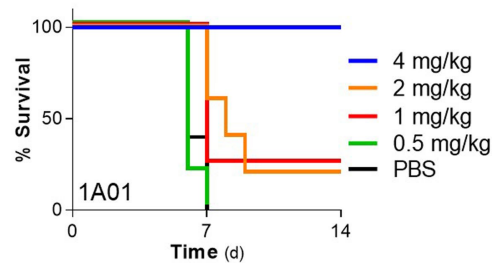

e

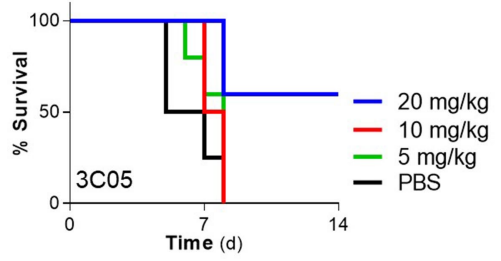

Extended Data Fig. 2 |Anti-HA and NA monoclonal antibody titration studies to determine the optimal dose required for protection against mouse influenza infection. Given the differential epitope specificities and in vitro neutralization potency of the selected anti-influenza monoclonal antibodies, titration studies were performed to assess the capacity of these antibodies to protect mice against lethal influenza infection. Anti-HA and NA antibodies (a, Fl6v3 ( $n=5$ mice per group for $8 \mathrm{mg} \mathrm{kg}^{-1}$ group, $n=6$ mice per group for all other groups); b, FY1 ( $n=6$ mice per group for 4 and $2 \mathrm{mg} \mathrm{kg}^{-1}, n=5$ mice per group for 1 and $0.5 \mathrm{mg} \mathrm{kg}^{-1}, n=4$ mice per group for PBS); $\mathbf{c}, 4 \mathrm{GO}$ ( $n=7$ mice per group for $0.5 \mathrm{mg} \mathrm{kg}^{-1}$ group, $n=6$ mice per group for all other groups); d, $1 \mathrm{A01}$ ( $n=4$ mice per group for $1 \mathrm{mg} \mathrm{kg}^{-1}$ group, $n=5$ mice per group for all other groups); e, $3 \mathrm{CO} 5$ ( $n=5$ mice per group for 20 and $5 \mathrm{mg} \mathrm{kg}^{-1}, n=4$ mice per group for $10 \mathrm{mg} \mathrm{kg}^{-1}$ and PBS groups)). All antibodies were expressed as human IgG1 and administered intraperitoneally (for FI6v3 and FY1) or intravenously (for 4G05,1A01, and 3C05) at the dose indicated to mice (C57BL/6) $4 \mathrm{~h}$ before lethal challenge with influenza (five LLD $_{50}$; H1N1 PR8 for a, b; H1N1 Neth/09 for $\mathbf{c}-\mathbf{e})$. Survival was monitored for 14 days. 
FI6v3 - Broadly (Group 1 and 2) neutralizing anti-HA stalk

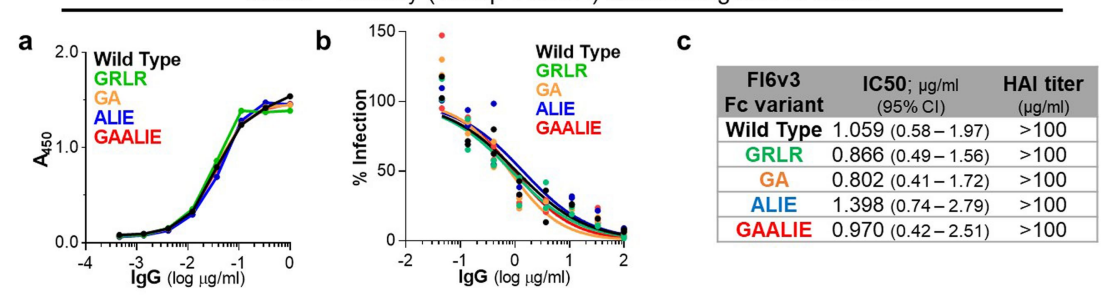

FY1 - Broadly (Group 1 and 2) neutralizing anti-HA stalk
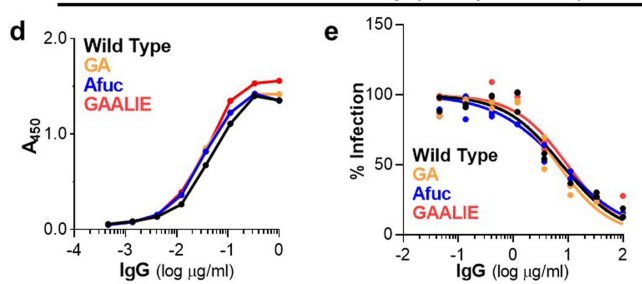

f

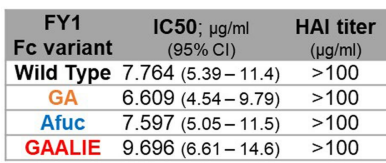

4G05 - Broadly (pan-H1) neutralizing anti-HA head

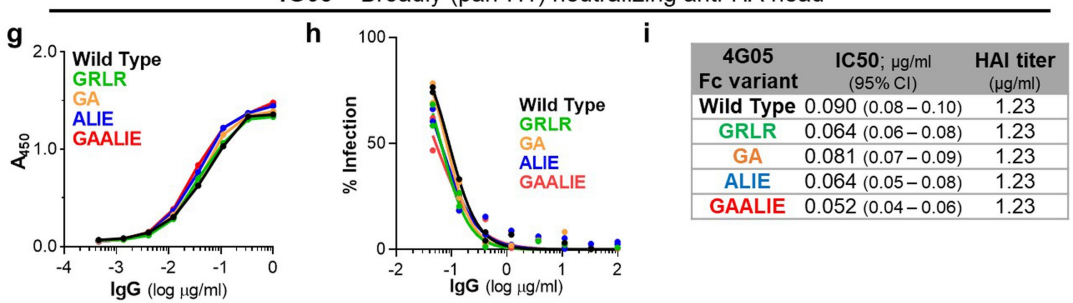

1 A01 - Broadly (pan-H1) binding, non-neutralizing anti-HA head
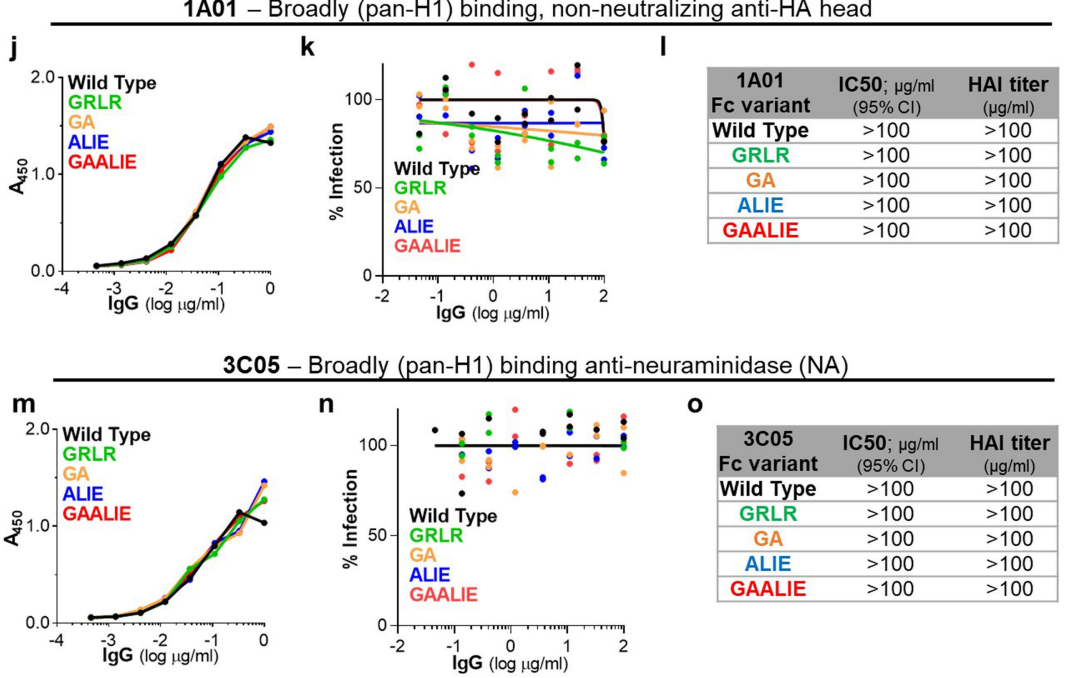

Extended Data Fig. 3 | In vitro characterization of the antigenic specificity, neutralization potency, and $\mathrm{HAI}$ activity of Fc domain variants of anti-influenza monoclonal antibodies. a-I, To study the role of $F c-F c \gamma R$ interactions in the antibody-mediated protection against influenza infection, Fc domain variants with differential $F c \gamma R$ affinities (Fig. 1b) were generated for antibodies that target distinct epitopes on influenza antigens (Fig. 1a). These antibodies include Fl6v3 (a-c) and FY1 (d-f), which both recognize the stalk region of influenza $\mathrm{HA}$ and exhibit broad and potent neutralizing activity against group 1 and 2 influenza strains, $4 \mathrm{GO} 5(\mathbf{g}-\mathbf{i})$, which is a pan- $\mathrm{H} 1$ monoclonal antibody against the globular head of HA and exhibits potent neutralizing and HAI activity, $1 \mathrm{AO} 1(\mathbf{j}-\mathbf{l})$, which is a pan-H1 anti-globular head HA antibody with no neutralizing or HAI activity, and $3 \mathrm{CO}$ ( $\mathbf{m}-\mathbf{o})$, a broadly

(pan-H1) protective antibody against NA. To confirm that changes in the Fc domain have no effect on the antigenic activity and Fab-mediated functions of these antibodies, Fc domain variants were characterized by $\operatorname{ELISA}(\mathbf{a}, \mathbf{d}, \mathbf{g}, \mathbf{j}, \mathbf{m}$; $n=1$ experiment performed in duplicates) to assess their specificity against purified HA (H1N1 PR8 strain for Fl6v3 and FY1; Cal/09 strain for 4G05, 1A01 and 3C05), by microneutralization assay (b,e, h, $\mathbf{k}$ and $\mathbf{n} ; \mathbf{c}, \mathbf{f}, \mathbf{i}, \mathbf{I}$ and $\mathbf{o}$ for $\mathrm{IC}_{50}$ values; $n=2$ independent experiments; data were fitted with nonlinear regression analysis (four-parameter) to calculate $\mathrm{IC}_{50}$ values) to evaluate their neutralizing activity against H1N1 (H1N1 PR8 strain for Fl6v3 and FY1; Neth/09 strain for 4G05, 1A01, and 3C05), and by HAI assay (H1N1 PR8 strain for FI6v3 and FY1; Neth/09 strain for 4G05,1A01, and $3 \mathrm{C} 05 ; n=2$ independent experiments) to determine their HAI titre (c, $\mathbf{f}, \mathbf{i}, \mathbf{l}$ and $\mathbf{o})$. 

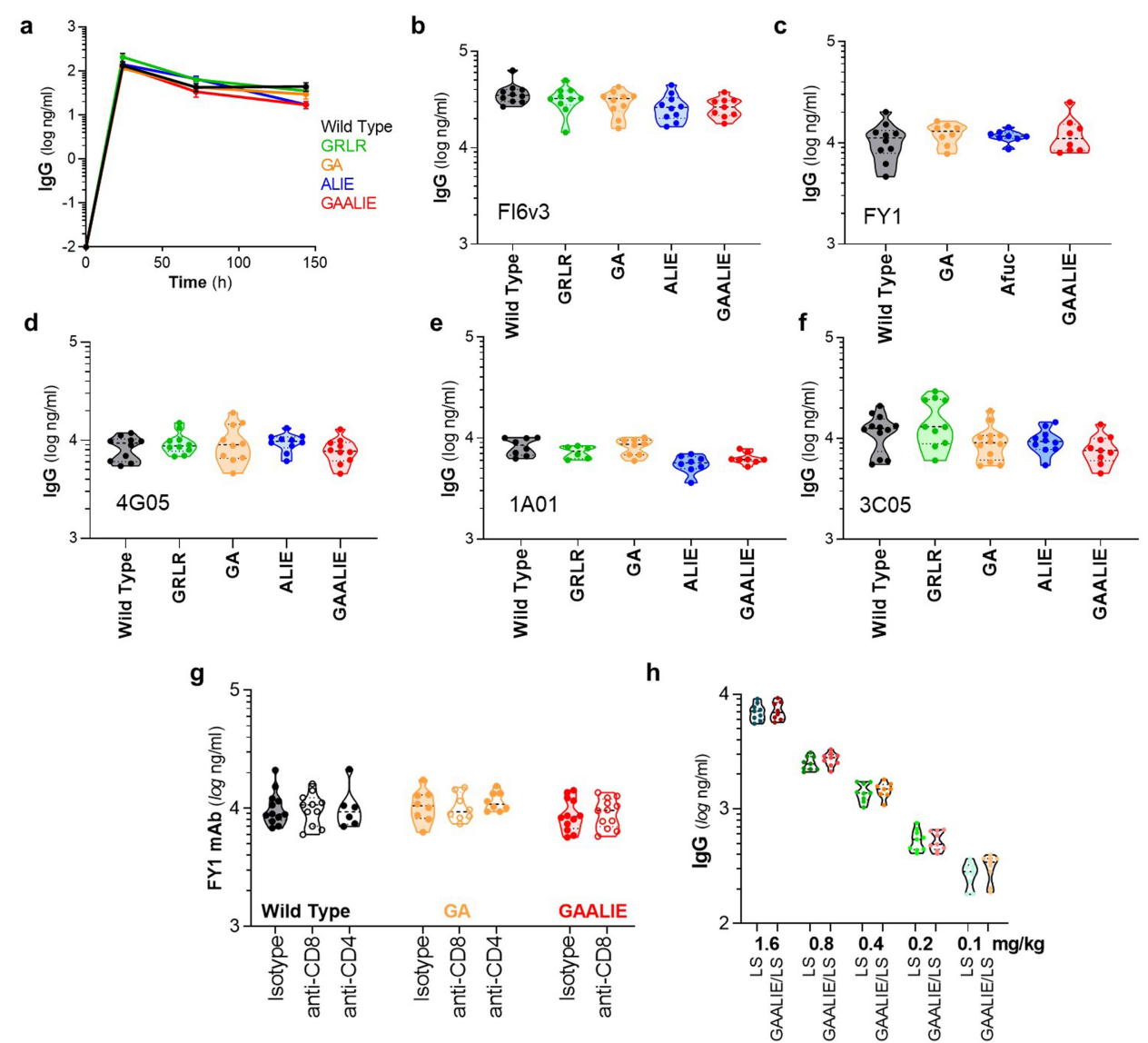

Extended Data Fig. 4 | In vivo half-life of Fc domain variants. a, Fc variants of an anti-HIV antibody (3BNC117) were administered (intravenously; $100 \mu \mathrm{g}$ ) to Fc $\gamma R$-humanized mice and antibody serum levels were determined by ELISA at various time points after antibody administration. $n=4$ mice per group in two independent experiments. Data are mean \pm s.e.m. b-f, To ensure that the observed differences in the protective activity of $\mathrm{Fc}$ variants of anti-HA and NA antibodies (Figs. 1 and 2) were not due to differential in vivo antibody half-lives, serum was obtained from influenza-infected mice (day 3 after infection) and analysed by ELISA to quantify antibody levels. (b: $n=9$ mice per group for GAALIE, $n=10$ mice per group for all other groups; $\mathbf{c}: n=10$ mice per group for wild type, $n=8$ mice per group for all other groups; $\mathbf{d}: n=10$ mice per group; $\mathbf{e}:$ $n=6$ mice per group for GRLR, $n=8$ mice per group for all other groups; $f: n=12$ mice per group for wild type and GA; $n=10$ mice per group for GRLR and ALIE; $n=9$ mice per group for GAALIE). $g$, To ensure that the observed differences in the protective activity of Fc variants of FY1 antibodies (Fig. 3e, f, Extended Data Fig.10d, e) were not due to differential in vivo antibody half-lives, serum was obtained from influenza-infected mice (day 3 after infection) and analysed by ELISA to quantify FY1 antibody levels. $n=12$ mice per group for wild type/ isotype, GAALIE/isotype, and GAALIE/anti-CD8 groups, $n=11$ mice per group for wild-type/anti-CD8, $n=8$ mice per group for GA/isotype, GA/anti-CD4, and $\mathrm{GA} /$ anti-CD4 groups, $n=6$ mice per group for wild-type/anti-CD4 group. $\mathbf{h}$, Titration studies were performed in a mouse model of antibody-mediated prophylaxis of influenza infection (Fig. 4d) and serum was obtained at the time of virus challenge and analysed by ELISA to quantify FY1 antibody levels. $n=10$ mice per group for LS $0.1 \mathrm{mg} \mathrm{kg}^{-1}$ dose, $n=8$ for GAALIE-LS at 1.6 and $0.4 \mathrm{mg} \mathrm{kg}^{-1}$ doses, $n=9$ mice per group for all other groups. 


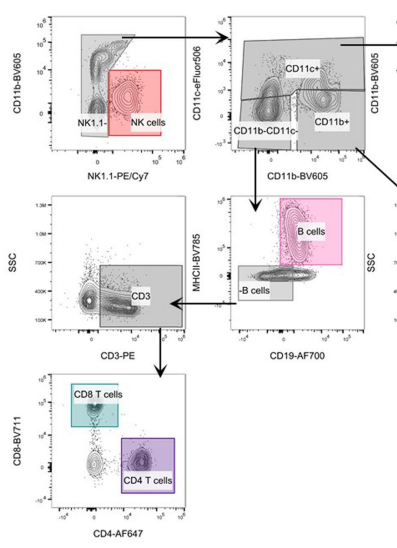

C
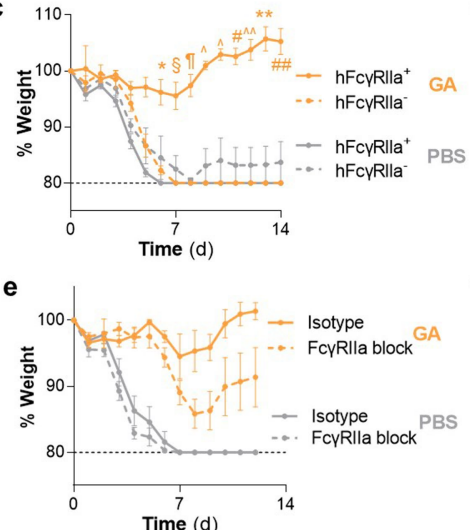

g
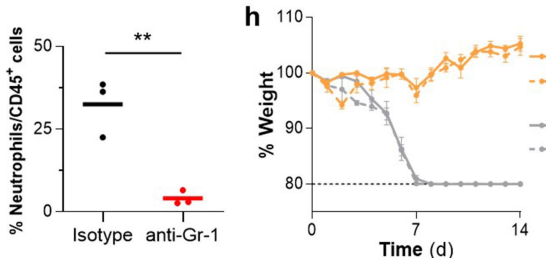

Extended Data Fig. 5 | Evaluation of the role of Fc $\gamma$ RIIa and the contribution of neutrophils in the antibody-mediated protection against influenza infection. a, b, To confirm the dependence of Fc $\gamma$ RIla engagement in driving the protective activity of the GA variant, mice expressing the human Fc $\gamma$ RIIa transgene on an $\mathrm{Fc}_{\mathrm{C}} \mathrm{R}_{\text {null }}$ background ( $\mathrm{hFc} \gamma \mathrm{RIIa}^{+}$; gating strategy (a) and representative flow cytometry histograms $(\mathbf{b})$ of Fc $\gamma$ RIIa expression in lung-resident leukocytes) or deficient for all classes of Fc $\gamma \mathrm{Rs}\left(\mathrm{hFc} \gamma \mathrm{RIIa}^{-}\right)$were administered with GA variants of FY1 (intraperitoneally $\left.2 \mathrm{mg} \mathrm{kg}^{-1}\right)(n=7$ mice per group for Fc $\gamma \mathrm{RIIa}^{+}, n=6$ mice per group for $\mathrm{Fc} \mathrm{RIIa}^{-}$in two independent experiments) or PBS ( $n=4$ mice per group for $\mathrm{Fc} \mathrm{RIIa}^{+}, n=5$ mice per group for Fc $\gamma \mathrm{RIIa}^{-}$in two independent experiments) $4 \mathrm{~h}$ before lethal challenge with influenza (H1N1; PR8, 5 mLD $_{50}$ ). c, d, Weight loss $(\mathbf{c})$ (mean \pm s.e.m.) and survival (d) were monitored for 14 days and compared by two-way ANOVA (Bonferroni post hoc analysis adjusted for multiple comparisons) $\left(\right.$ c: ${ }^{*} P=0.02 \S P=0.03$, $\Phi P=0.006,{ }^{\wedge} P<0.0001, \# P=0.0002,{ }^{\wedge} \wedge P=0.0006,{ }^{* *} P=0.001, \# \# P=0.002$ versus GA-treated Fc $\gamma \mathrm{RIIa}^{-}$) and log-rank (Mantel-Cox) test, respectively (d: ${ }^{*} P=0.0006$ versus GA-treated Fc $\left.\gamma \mathrm{RIIa}^{-}\right)$. e, f, Fc $\gamma \mathrm{R}$-humanized mice were administered with GA variants of Fl6v3 (intraperitoneally $4 \mathrm{mg} \mathrm{kg}^{-1}$ ) before challenge with PR8 (as described in c, d). To block the ligand binding activity of Fc $\gamma$ RIIa, recombinant anti-Fc $\gamma$ RIIa (clone IV.3) expressed as human IgG1 GRLR b

d

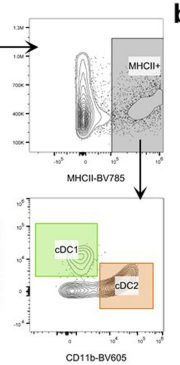

o

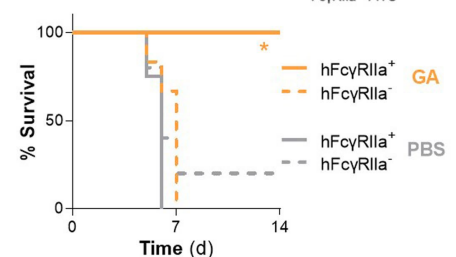

f

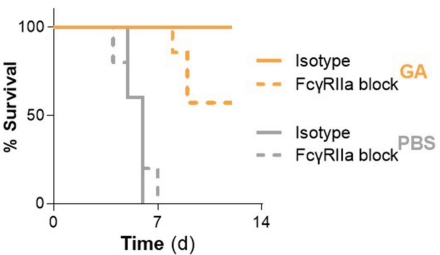

i

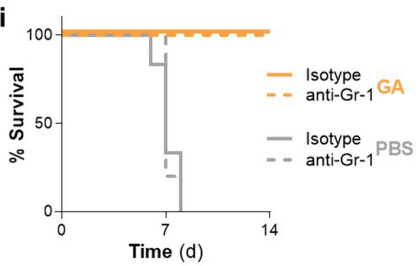

variant to abrogate $\mathrm{Fc} \gamma \mathrm{R}$ binding or isotype control (anti-hapten (NP) monoclonal antibody; clone $3 \mathrm{C} 13)$ was administered intranasally $(80 \mu \mathrm{g})$ to mice 1 day after virus challenge ( $n=7$ mice per group for GA/IV.3-treated group, $n=5$ mice per group for all other groups in two independent experiments) and weight loss (e) (mean \pm s.e.m.) and survival (f) were monitored.g, To establish the efficiency of antibody-mediated neutrophil depletion, Fc $\gamma$ R-humanized mice ( $n=3$ mice per group in one experiment) were injected intravenously with $150 \mu \mathrm{g}$ anti-mouse $\mathrm{Gr}-1$ monoclonal antibody (clone RB6-8C5) or isotype control (clone LTF-2). The abundance of neutrophils $\left(\mathrm{Ly}_{6 \mathrm{G}}{ }^{+}\right)$in peripheral blood was determined 2 days after antibody administration by flow cytometry. ${ }^{* *} P=0.0053$, two-sided unpaired $t$-test. $\mathbf{h}$, $\mathbf{i}$, To assess the contribution of neutrophils to the protective activity of Fc $\gamma$ RIIa-enhanced variants of anti-influenza antibodies, GA variants of the anti-HA stalk antibody FY1 were administered intraperitoneally $\left(2 \mathrm{mg} \mathrm{kg}^{-1}\right)$ to Fc $\gamma \mathrm{R}$-humanized mice $(n=6$ mice per group, except for PBS/Gr-1 treated ( $n=5$ mice per group) in two independent experiments) $4 \mathrm{~h}$ before lethal challenge with PR8. Isotype or anti-mouse $\mathrm{Gr}-1$ monoclonal antibodies ( $150 \mu \mathrm{g}$ intravenously) were administered on day 1 after infection and weight loss (h) (mean \pm s.e.m.) and survival (i) were recorded. 
a
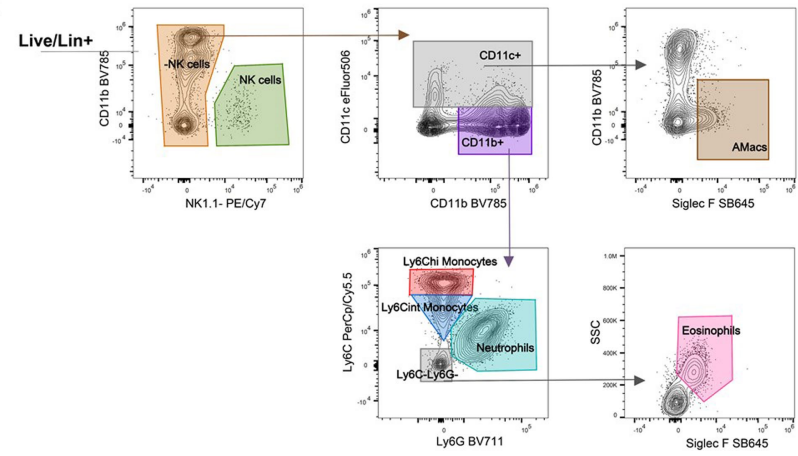

b
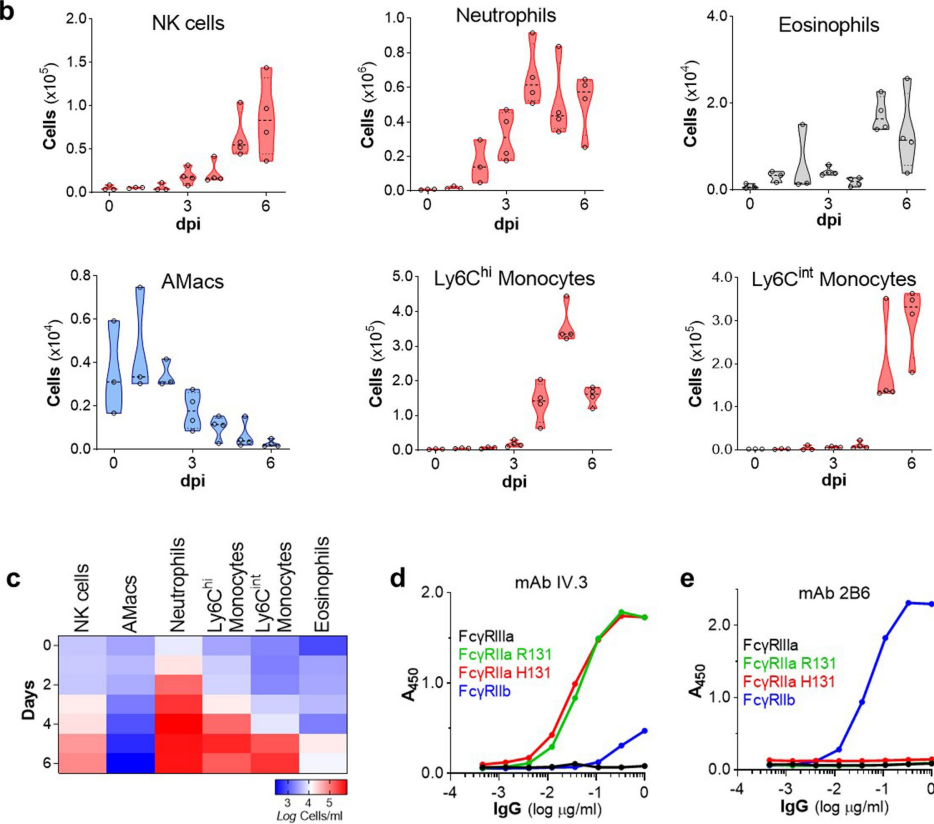

f
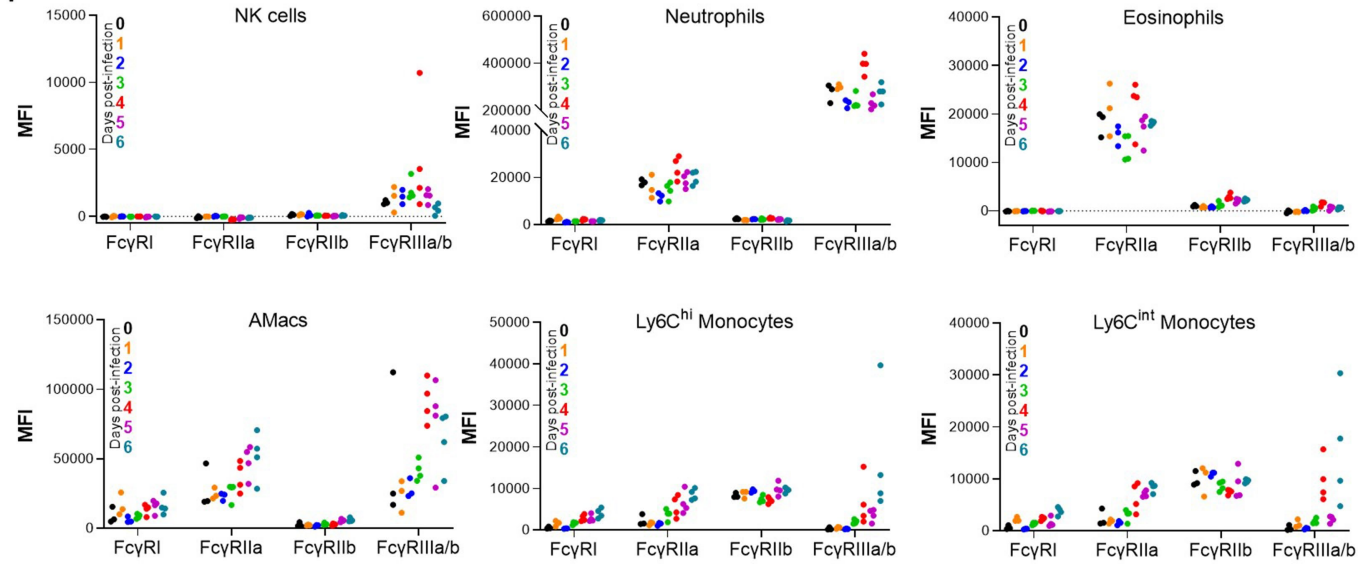

Extended Data Fig. 6 | Abundance and FcyR expression profile of leukocyte populations in the lungs of influenza-infected FcyR-humanized mice at different time points afteer infection. a-f, To determine the abundance and Fc $\gamma R$ expression profile of lung resident and infiltrated leukocytes during the course of influenza infection, cohorts of Fc $\gamma \mathrm{R}$-humanized mice were infected (intranasally with $\mathrm{H} 1 \mathrm{~N} 1 \mathrm{PR} 8 ; 5 \mathrm{mLD}_{50}$ ) and euthanized at different time points after infection (day 0 to 6 ). Lungs were homogenized and analysed by flow cytometry (a: gating strategy) to determine the frequency (b, c) and Fc $\gamma \mathbf{R}$ expression profile (f) of innate effector leukocytes. $n=3$ and $n=4$ mice per time point for day 0-2 and day 3-6 time points, respectively. Influenza infection was associated with the recruitment of natural killer (NK) cells, neutrophils and monocytes, whereas the number of alveolar macrophages was reduced at the later stages of infection. Owing to the high degree of sequence similarity between Fc $\gamma$ RIIa and Fc $\gamma R$ RIIb, expression of these Fc $\gamma R$ s was assessed using antibody clones (d: IV.3 for Fc $\gamma$ RIIa; e: $2 \mathrm{~B} 6$ for Fc $\gamma R$ RIIb) that exhibit high specificity, as assessed by ELISA using recombinant $\mathrm{Fc} \gamma \mathrm{Rs}$ ( $n=1$ experiment performed in duplicates). Analysis of the Fc $\gamma$ R expression profile (MFI) revealed that influenza infection had no effect on the levels of Fc $\gamma$ Rs expressed by the various leukocyte types. With the exception of natural killer cells, which expressed only FcyRIIIa, most innate effector leukocytes co-expressed multiple Fc $\gamma R$ s, including the activating Fc $\gamma R s$, Fc $\gamma R$ IIla and Fc $\gamma R$ IIIa/b, as well as the inhibitory Fc $\gamma$ RIIb. $n=3$ and $n=4$ mice per time point for day $0-2$ and day 3-6 time points, respectively. 
a
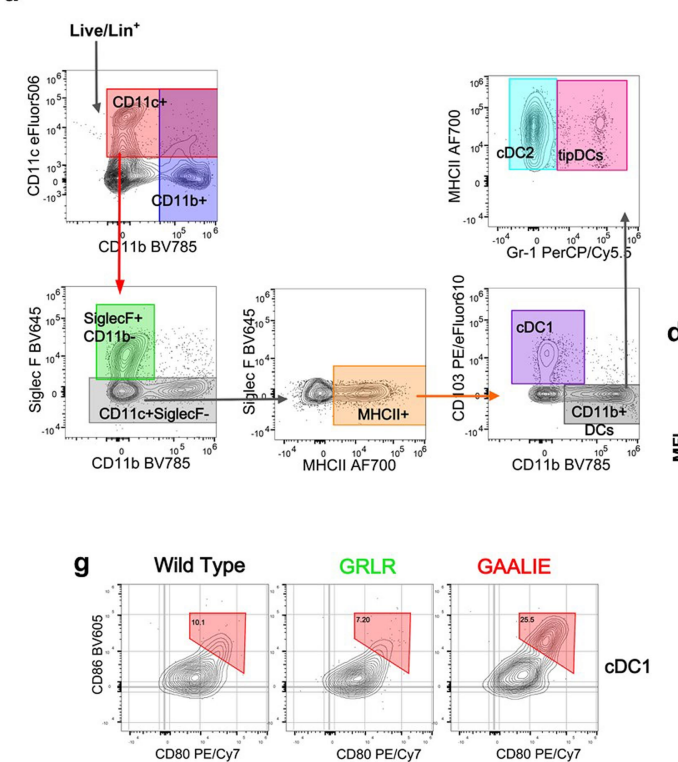

h

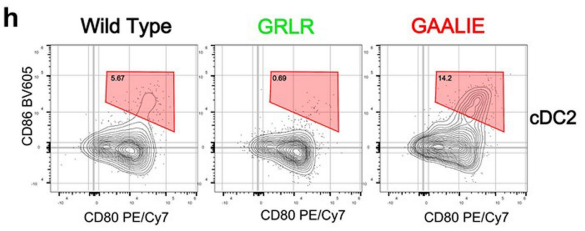

b

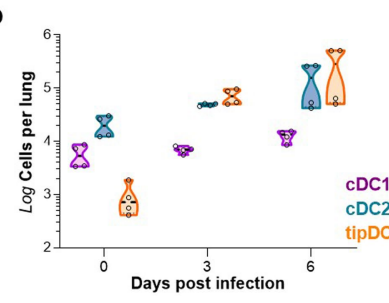

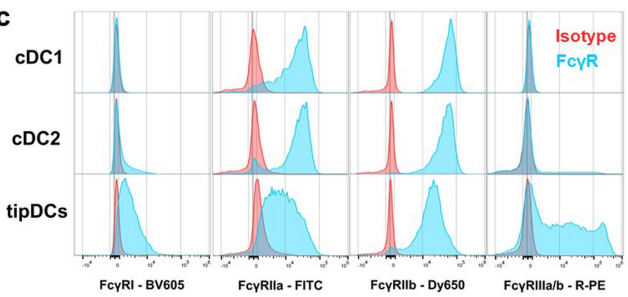

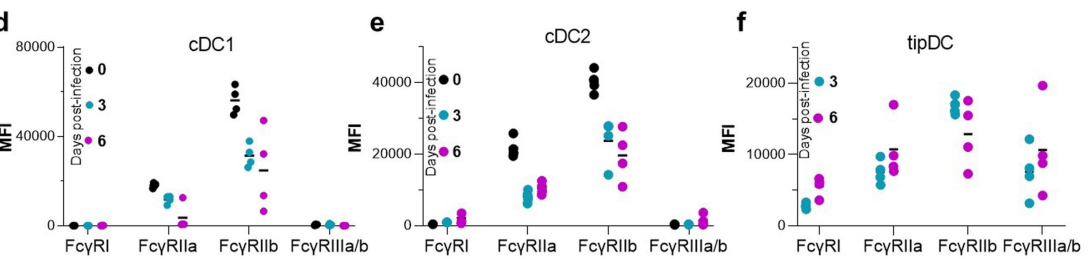
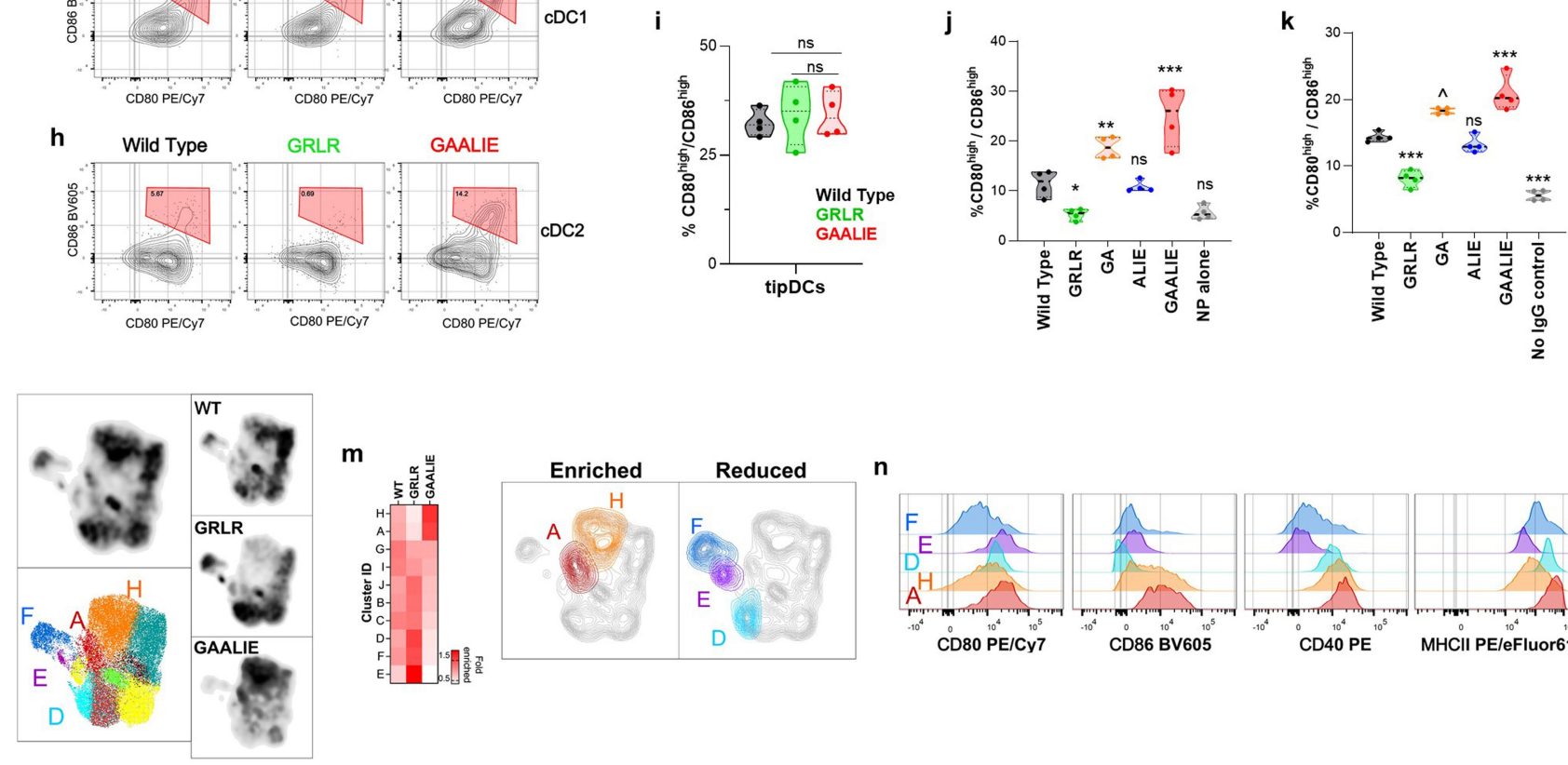

Extended Data Fig. 7 |See next page for caption. 
Extended Data Fig. 7 | Treatment of Fc $\gamma$ R-humanized mice with GAALIE variants of anti-HA antibodies is associated with increased frequency of activated dendritic cells. a-f, To determine the abundance and Fc $\gamma R$ expression profile of dendritic cell subsets during the course of influenza infection, cohorts of Fc $\gamma R$-humanized mice were infected (intranasally with $\mathrm{H} 1 \mathrm{N1}$ PR8; $5 \mathrm{mLD}_{50}$ ) and euthanized at different time points after infection (day 0 to 6). Lungs were homogenized and analysed by flow cytometry (a: gating strategy) to determine the frequency (b) and $F c \gamma R$ expression profile (c: representative flow cytometry overlay; d-f: MFI) of the three major dendritic cell subsets identified: $\mathrm{cDC1}, \mathrm{cDC} 2$ and tipDCs. Influenza infection was not associated with any major changes in the number of lung-resident CDC1 and cDC2, whereas tipDCs were almost absent at baseline, but their number increased markedly after infection. $\mathrm{cDC} 1$ and $\mathrm{cDC} 2$ expressed Fc $\gamma \mathrm{RIIa}$ and Fc $\mathrm{R}$ RIIb, but they were negative for Fc $\gamma$ RIIIa. By contrast, tipDCs expressed Fc $\gamma$ RIIa and Fc $\gamma$ RIIIa, along with the inhibitory Fc $\gamma$ RIIb. Owing to the very low number of tipDCs at baseline, Fc $\gamma$ R expression (MFI) was omitted. $n=4$ mice per time point assessed. $\mathbf{g}-\mathbf{i}$, To investigate the effect of enhanced Fc $\gamma$ RIIa engagement by GAALIE variants on the maturation status of dendritic cells, Fc $\mathrm{R}$-humanized mice were treated with Fc domain variants of the anti-HA stalk antibody FI6v3, exhibiting differential FcyR affinity-wild type IgG1 (baseline Fc $\gamma$ R affinity), GRLR (diminished binding to all classes of Fc $\gamma$ Rs), and GAALIE (increased $F c \gamma$ RIIa and Fc $\gamma$ RIIIa affinity). Fc domain variants were administered intraperitoneally $\left(3 \mathrm{mg} \mathrm{kg}^{-1}\right)$ to Fc $\gamma \mathrm{R}$-humanized mice $(n=4$ mice per treatment group in two independent experiments) $4 \mathrm{~h}$ before lethal challenge with H1N1 (PR8; $5 \mathrm{mLD}_{50}$ ). Mice were euthanized on day 4 and lung-resident dendritic cells were analysed by flow cy tometry. The abundance of mature (defined as CD80 $\left.{ }^{\text {high }} \mathrm{CD} 86^{\text {high }}\right) \mathrm{cDC} 1(\mathbf{g}), \mathrm{cDC} 2(\mathbf{h})$, and tipDCs (i) was compared between mice treated with the various Fc domain variants of FI6v3. Representative flow cytometry plots from data presented in Fig. 3a. In contrast to cDC1 and cDC2, no differences were observed in the maturation status of tipDCs among mice treated with different FI6v3 Fc variants (one-way ANOVA). j, k, In vitro differentiated monocyte-derived dendritic cells ( $n=4$ peripheral blood mononuclear cell donors performed in two independent experiments) were stimulated overnight with IgG immune complexes (anti-NP-NP-BSA immune complexes (j) and heat-aggregated IgG complexes $(\mathbf{k}))$. The abundance (percentage) of mature dendritic cells (defined as CD80 ${ }^{\text {high }} \mathrm{CD} 86^{\text {high }}$ ) was assessed by multicolour flow cytometry and compared against the corresponding wild-type-treated group by one-way ANOVA (Bonferroni post hoc analysis adjusted for multiple comparisons) ${ }^{*} P=0.0417,{ }^{* *} P=0.0134$, ${ }^{* * *} P<0.0001, \wedge P=0.0049$. I-n, Cluster analysis of dendritic cell populations present in the lungs of influenza-infected mice treated with Fc domain variants of anti-HA monoclonal antibodies. Mice $(n=4$ mice per group in two independent experiments) were treated as described in $\mathbf{g}-\mathbf{i}$, euthanized on day 4 after infection and dendritic cells $\left(\mathrm{Lin}^{+} \mathrm{MHCII}{ }^{+} \mathrm{CD} 11 \mathrm{c}^{+}\right.$) were analysed by multicolour flow cytometry. Data were dimensionally reduced and visualized using the UMAP algorithm. I, UMAP plots of dendritic cells in mice treated with the different Fc domain variants are presented. Populations were identified by X-shift using KNN density estimation and assigned IDs (A-J). $\mathbf{m}$, The abundance of the various dendritic cell clusters in the different treatment groups was plotted and populations that are enriched or reduced in GAALIE-treated mice were identified.n, Histogram plots of the expression of CD80, CD86, CD40 and $\mathrm{MHCII}$ in dendritic cell populations that are enriched (red, A; and orange, $\mathrm{H}$ ) or reduced (cyan, D; purple, E; and blue, F) in GAALIE-treated mice. GAALIE treatment was associated with the enrichment of dendritic cell populations characterized by high levels of CD86 and CD40 expression. Results are from four mice per treatment condition in two independent experiments. 


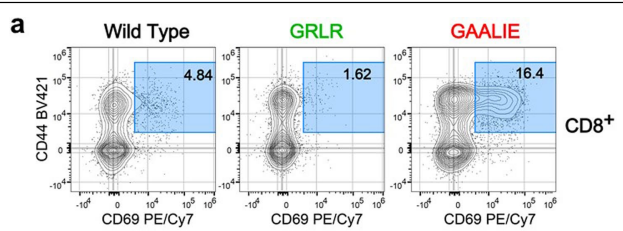

b

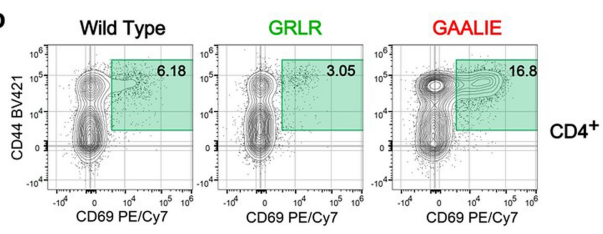

Extended Data Fig. 8 | Treatment of Fc $\gamma$ R-humanized mice with GAALIE variants of anti-HA stalk antibodies is associated with increased activation

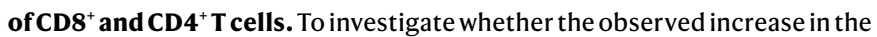
frequency of mature dendritic cells in mice treated with GAALIE variants of anti-HA monoclonal antibodies was associated with enhanced $\mathrm{T}$ cell responses, the activation status of $\mathrm{CD} 8^{+}$and $\mathrm{CD} 4^{+} T$ cells was analysed and compared between mice treated with anti-HA Fc domain variants with differential $\mathrm{Fc} \gamma \mathrm{R}$ affinity (wild-type IgG1, GRLR and GAALIE). Fc domain variants of the anti-HA stalk antibody Fl6v3 were administered (intraperitoneally $3 \mathrm{mg} \mathrm{kg}^{-1}$ ) to Fc $\gamma \mathrm{R}$-humanized mice before lethal challenge with H1N1 (PR8; $\left.5 \mathrm{mLD}_{50}\right)$. Mice ( $n=4$ mice per group in two independent experiments) were euthanized on day 4 after infection and $T$ cell populations were analysed by multicolour flow cytometry. a, b, The frequency of activated (defined as $\left.\mathrm{CD} 44^{\mathrm{hi}} \mathrm{CD} 69^{+}\right) \mathrm{CD} 8^{+}(\mathbf{a})$ and $\mathrm{CD}^{+}(\mathbf{b}) \mathrm{T}$ cells was compared between mice treated with the various $\mathrm{Fc}$ domain variants of Fl6v3. Representative flow cytometry plots from data in c

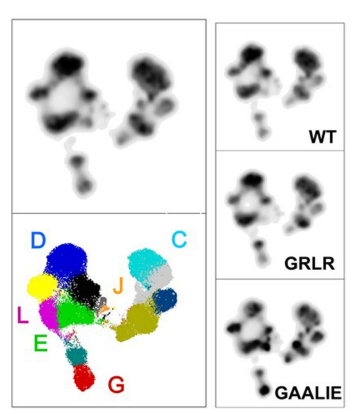

e

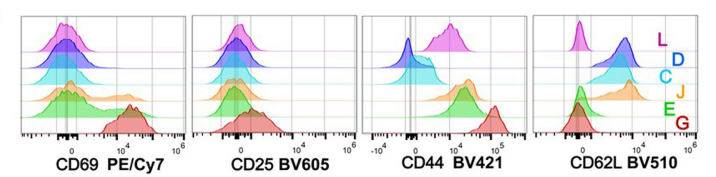

d

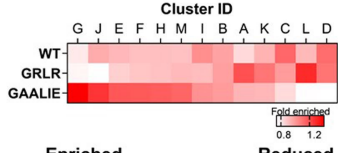

Enriched Reduced

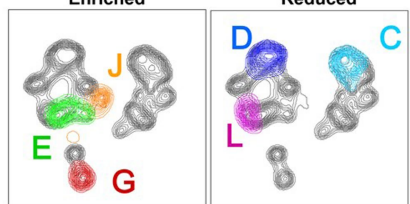

Fig. 3c. In addition, cluster analysis of T cell populations present in the lungs of influenza-infected mice treated with Fc domain variants of anti-HA monoclonal antibodies was performed. Flow cytometry data were dimensionally reduced and visualized using the UMAP algorithm. c, UMAP plots of T cells in mice treated with the different $\mathrm{Fc}$ domain variants are presented. Populations were identified by X-shift using KNN density estimation and assigned IDs (A-M). d, Heat map of the abundance of the various $T$ cell clusters in the different treatment groups. Populations that are enriched or reduced in GAALIE-treated mice were identified.e, Histogram plots of the expression of CD69, CD25, CD44 and CD62L in T cell populations that are enriched (red, G; green, E; orange,J) or reduced (cyan, C; blue, D; magenta, L) in GAALIE-treated mice. GAALIE treatment was associated with the enrichment of $T$ cell populations characterized by high levels of CD69, CD44 and CD25 expression. Results are from four mice per treatment condition. 


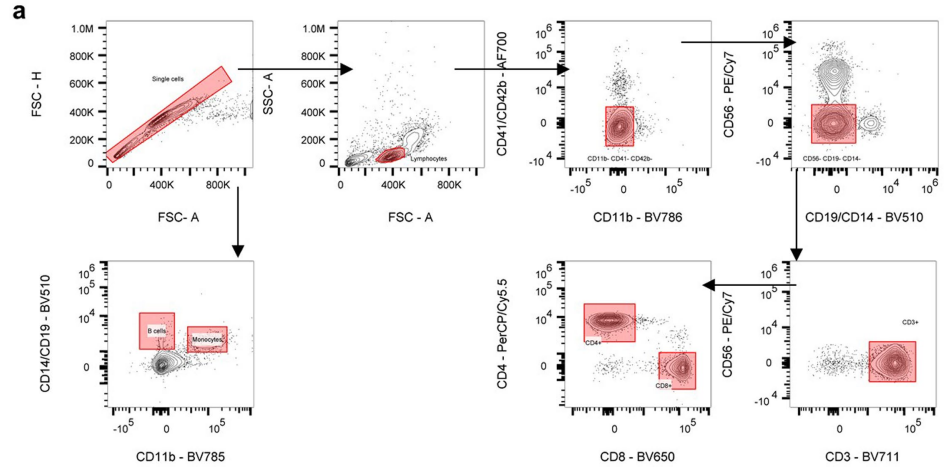

b

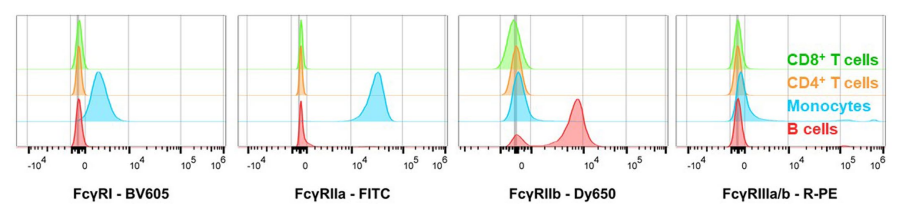

c

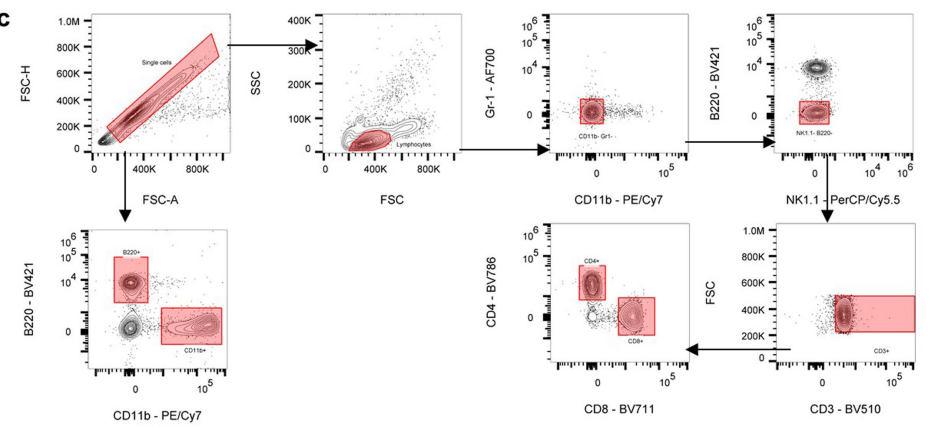

d
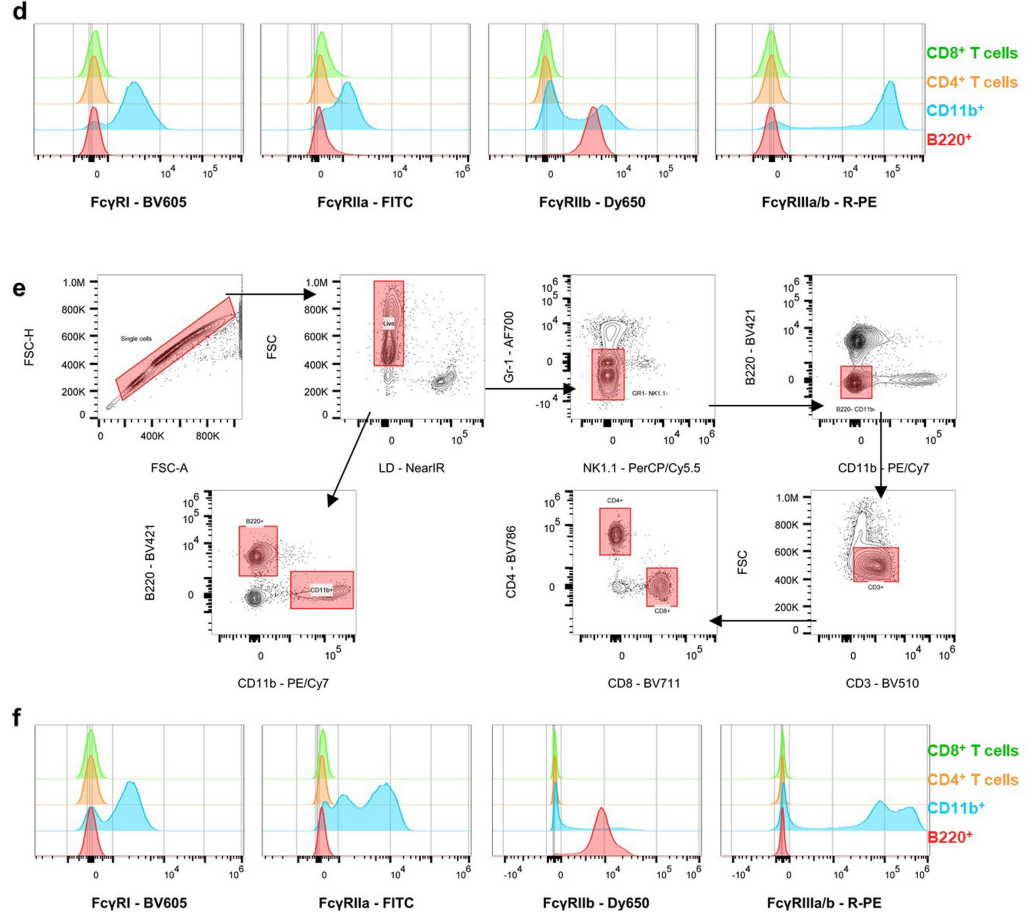

g

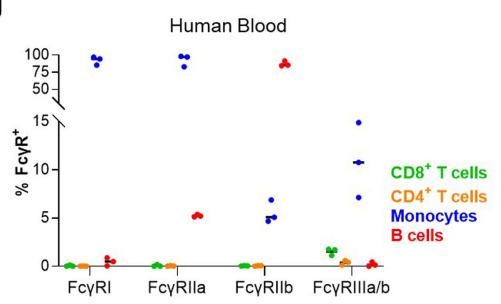

h
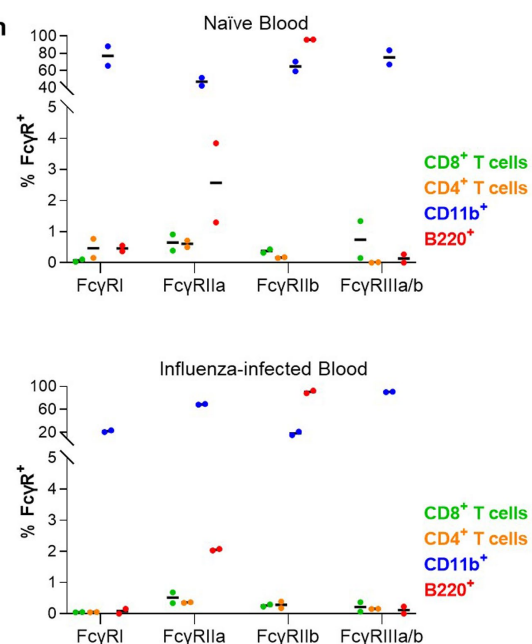

j $\left.\begin{array}{r}100 \\ 75 \\ 50\end{array}\right] \div \quad$ Naĩve Spleen $\infty$
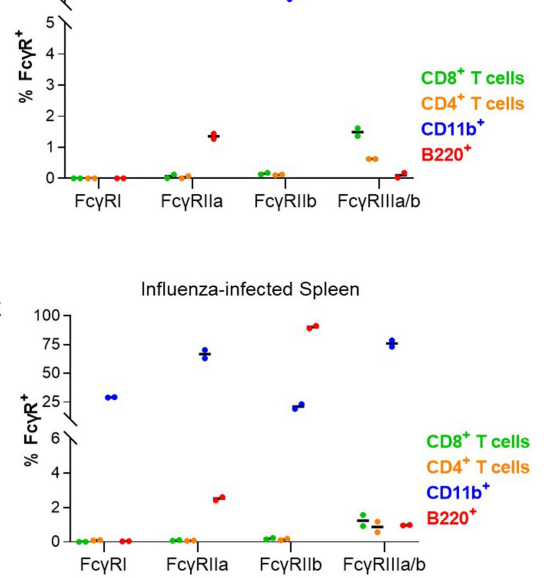

I

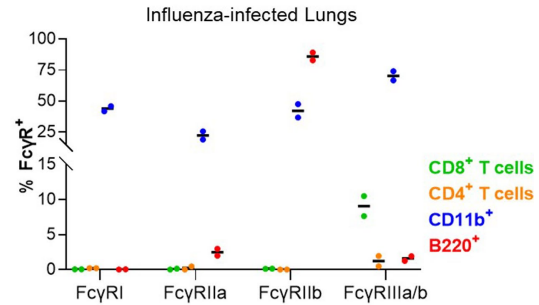

Extended Data Fig. 9 |See next page for caption. 


\section{Article}

Extended Data Fig. 9|FcyR expression analysis of T cells. To determine whether human $\mathrm{T}$ cells express human $\mathrm{F} c \gamma \mathrm{Rs}$, human peripheral blood mononuclear cells were analysed by multicolour flow cytometry. a, Gating strategy for identifying human $\mathrm{T}$ cells. Monocytes $\left(\mathrm{CD} 14^{+} \mathrm{CD} 11 \mathrm{~b}^{+}\right)$and $\mathrm{B}$ cells $\left(\mathrm{CD} 19^{+} \mathrm{CD} 1 \mathrm{~b}^{-}\right)$were included as controls for $\mathrm{Fc} \gamma \mathrm{R}$ immunofluorescence staining. b, $\mathbf{g}$, Analysis of Fc $\gamma$ R expression revealed that human T cells are negative for $\mathrm{Fc} \gamma \mathrm{R}$ expression (b: representative flow cytometry histogram overlays; g: quantification of $\mathrm{Fc} \gamma \mathrm{R}^{+}$cells; $\boldsymbol{n}=3$ donors). $\mathbf{c}-\mathbf{f}, \mathbf{h}-\mathbf{l}$, Human $\mathrm{Fc} \gamma \mathrm{R}$ expression on peripheral blood $(\mathbf{c}, \mathbf{d}, \mathbf{h}, \mathbf{i})$, spleen $(\mathbf{e}, \mathbf{f}, \mathbf{j}, \mathbf{k})$, or lung $(\mathbf{I}) \mathrm{T}$ cells from naive $(\mathbf{h}, \mathbf{j})$ or influenza-infected $\left(\mathbf{i}, \mathbf{k}, \mathbf{l}\right.$; day 6 after infection $\left(5 \mathrm{mLD}_{50}\right.$; H1N1 PR8)) Fc $\gamma$ R-humanized mice was assessed by multicolour flow cytometry.
Gating strategy for identifying $\mathrm{T}$ cells in mouse blood (c), spleen or lungs (e). Myeloid cells $\left(\mathrm{CD} 11 \mathrm{~b}^{+}\right)$and B cells $\left(\mathrm{B} 22 \mathrm{O}^{+}\right)$were included as controls for $\mathrm{Fc} \gamma \mathrm{R}$ immunofluorescence staining. Analysis of $\mathrm{Fc} \gamma \mathrm{R}$ expression on $\mathrm{T}$ cells from naive or influenza-infected FcyR-humanized mice revealed minimal expression of $\mathrm{Fc} \gamma \mathrm{Rs}$ on $\mathrm{T}$ cell (d, $\mathbf{f}$ : representative flow cytometry histogram overlays; h-l: quantification of Fc $\gamma \mathrm{R}^{+}$cells ( $n=2$ mice per group in one experiment) in the $\operatorname{blood}(\mathbf{h}, \mathbf{i})$, spleen $(\mathbf{j}, \mathbf{k})$ and lungs (l) of naive or influenza-infected mice). Although a small fraction of $B$ cells appears positive for $F c \gamma R$ RIa expression, this is probably due to the cross-reactivity of the anti-FcyRIIa antibody (clone IV.3) to FcyRIIb (see Extended Data Fig. 6d). 

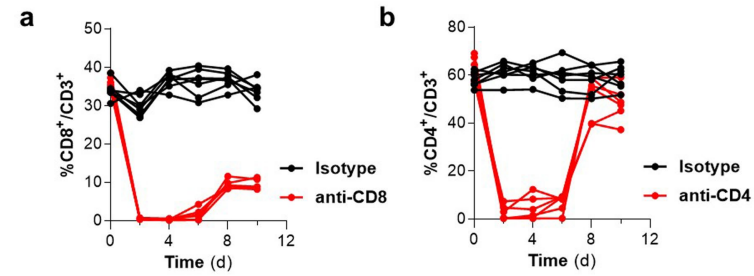

C
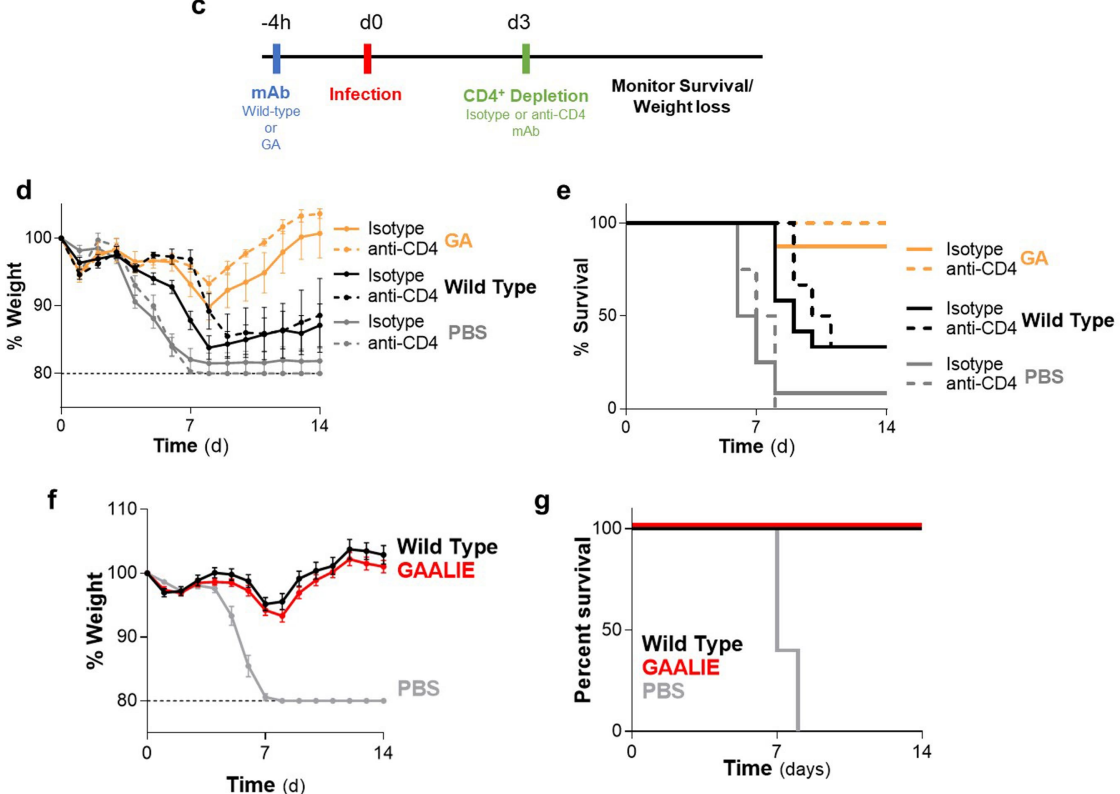

g
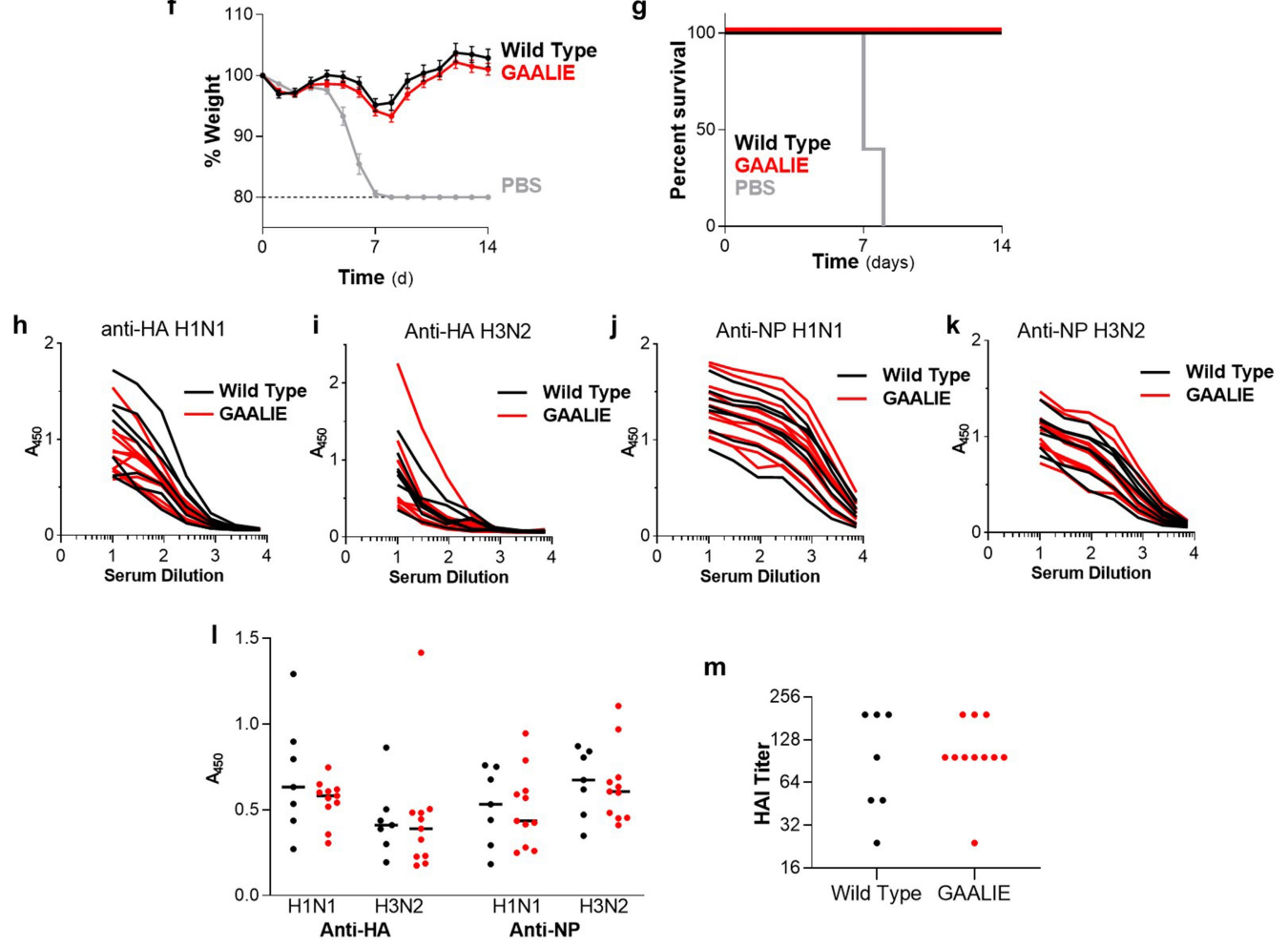

m

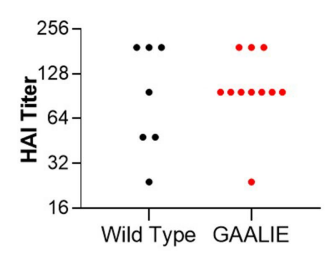

Extended Data Fig. 10 |See next page for caption. 


\section{Article}

Extended Data Fig. $10 \mid$ T cell depletion experiments and the effect of Fc $\gamma$ RIIa-enhanced variants on anti-influenza IgG responses. $a, b$, To establish the efficiency of antibody-mediated $\mathrm{CD} 8^{+}$or $\mathrm{CD} 4^{+} \mathrm{T}$ cell depletion, Fc $\gamma \mathrm{R}$-humanized mice were injected intravenously with $150 \mu \mathrm{g}$ anti-mouse CD8 $\alpha$ monoclonal antibody (clone 2.43) or anti-mouse CD4 (clone GK1.5), or isotype control (clone LTF-2). The abundance of $\mathrm{CD}^{+}(\mathbf{a})$ or $\mathrm{CD}^{+}(\mathbf{b}) \mathrm{T}$ cells in peripheral blood was determined at various time points after antibody administration by flow cytometry. Baseline $\mathrm{CD} 8^{+}$or $\mathrm{CD} 4^{+} \mathrm{T}$ cell frequencies were determined in blood samples obtained before antibody administration. Results are expressed as the percentage of $\mathrm{CD} 8^{+} \mathrm{CD} 3^{+}$or $\mathrm{CD} 4^{+} \mathrm{CD} 3^{+} \mathrm{T}$ cells $(n=6$ for anti-CD8 and anti-CD4-treated groups, $n=7$ mice per group for isotype groups in two independent experiments).c-e, To determine the contribution of $\mathrm{CD}^{+} \mathrm{T}$ cells in the protective activity of Fc $\gamma \mathrm{RIIa-enhanced} \mathrm{variants} \mathrm{of} \mathrm{anti-}$ influenza monoclonal antibodies, $\mathrm{CD}^{+}{ }^{+}$depletion studies were performed. c, Wild-type or GA variants of the anti-HA stalk antibody FY1 were administered intraperitoneally $\left(2 \mathrm{mg} \mathrm{kg}^{-1}\right)$ to Fc $\mathrm{R}$-humanized mice $4 \mathrm{~h}$ before lethal challenge with influenza $\left(5 \mathrm{mLD}_{50} ; \mathrm{H} 1 \mathrm{~N} 1 \mathrm{PR} 8\right)$. Isotype or anti-mouse CD4 antibodies ( $150 \mu \mathrm{g}$ intravenously) were administered on day 3 after infection.
Isotype-treated groups: $n=12$ mice per group for wild-type and PBS groups $n=8$ mice per group for GA group; anti-CD4-treated groups: $n=6$ mice per group for wild type, $n=8 \mathrm{mice} /$ group for $\mathrm{GA}, n=4$ mice per group for $\mathrm{PBS}$ in two independent experiments. d, e, Weight loss (d) (mean \pm s.e.m.) and survival (e) were monitored for 14 days. Data for isotype-treated mice are also in Fig. $3 e$, $\mathrm{f}$. f-m, Wild-type or GAALIE Fc variants for the anti-HA globular head antibody $1 \mathrm{A0} 01$ were administered (intravenously $4 \mathrm{mg} \mathrm{kg}^{-1}$ ) to Fc $\gamma \mathrm{R}$-humanized mice $(n=7$ mice per group for wild-type group, $n=11$ mice per group for GAALIE-treated group, $n=5$ mice per group for PBS-treated in two independent experiments) $4 \mathrm{~h}$ before lethal challenge with influenza $\left(5 \mathrm{mLD}_{50} ; \mathrm{H1N1}\right.$ Neth/09).f, g, Weight loss (mean \pm s.e.m.) and survival (g) were monitored for 14 days. $\mathbf{h}-\mathbf{k}$, IgG responses against $\mathrm{HA}(\mathbf{h}, \mathbf{i})$ and $\mathrm{NP}(\mathbf{j}, \mathbf{k})$ from homologous $(\mathrm{H} 1 \mathrm{~N} 1 ; \mathrm{A} / \mathrm{California/}$ 04/2009) or heterologous (H3N2; A/x31) strains were evaluated in surviving mice. I, Analysis of IgG titres against homologous or heterologous HA or NP revealed no differences among mice previously treated with wild-type or GAALIE Fc variants of 1A01 antibody. $\mathbf{m}$, Similarly, comparable HAI titres were noted among the treatment groups. 


\section{Reporting Summary}

Nature Research wishes to improve the reproducibility of the work that we publish. This form provides structure for consistency and transparency in reporting. For further information on Nature Research policies, see Authors \& Referees and the Editorial Policy Checklist.

\section{Statistics}

For all statistical analyses, confirm that the following items are present in the figure legend, table legend, main text, or Methods section.

n/a Confirmed

$\square \bigotimes$ The exact sample size $(n)$ for each experimental group/condition, given as a discrete number and unit of measurement

$\square$ \A statement on whether measurements were taken from distinct samples or whether the same sample was measured repeatedly

The statistical test(s) used AND whether they are one- or two-sided

$\triangle$ Only common tests should be described solely by name; describe more complex techniques in the Methods section.

$\triangle \square$ A description of all covariates tested

$\square \bigotimes$ A description of any assumptions or corrections, such as tests of normality and adjustment for multiple comparisons

X A full description of the statistical parameters including central tendency (e.g. means) or other basic estimates (e.g. regression coefficient) X $\square$ AND variation (e.g. standard deviation) or associated estimates of uncertainty (e.g. confidence intervals)

$\varnothing$ For null hypothesis testing, the test statistic (e.g. $F, t, r$ ) with confidence intervals, effect sizes, degrees of freedom and $P$ value noted

Give P values as exact values whenever suitable.

Х $\square$ For Bayesian analysis, information on the choice of priors and Markov chain Monte Carlo settings

$\bigotimes \square$ For hierarchical and complex designs, identification of the appropriate level for tests and full reporting of outcomes

Х Estimates of effect sizes (e.g. Cohen's $d$, Pearson's $r$ ), indicating how they were calculated

our web collection on statistics for biologists contains articles on many of the points above.

\section{Software and code}

Policy information about availability of computer code

Data collection GE Unicorn v6.3, SoftMaxPro v7.0.2, Biacore T200 control software v2.0, Attune NxT flow cytometry software v3.1.2, Microsoft Excel V2008

Data analysis

UCSF ChimeraX v0.94, GE Unicorn v6.3, SoftMaxPro v7.0.2, Biacore T200 evaluation software v2.0, Graphpad Prism v8.4, Microsoft Excel v2008, SeqBuilder Pro v16 and v17, SeqMan Pro v16 and v17, FlowJo v10.6.2 with the following plugins: Downsample v3.2, UMAP v2.2, and X-shift v1.3

For manuscripts utilizing custom algorithms or software that are central to the research but not yet described in published literature, software must be made available to editors/reviewers. We strongly encourage code deposition in a community repository (e.g. GitHub). See the Nature Research guidelines for submitting code \& software for further information.

\section{Data}

Policy information about availability of data

All manuscripts must include a data availability statement. This statement should provide the following information, where applicable:

- Accession codes, unique identifiers, or web links for publicly available datasets

- A list of figures that have associated raw data

- A description of any restrictions on data availability

Raw data for all main and extended data figures are included in the manuscript as source files. 


\section{Field-specific reporting}

Please select the one below that is the best fit for your research. If you are not sure, read the appropriate sections before making your selection. $\bigotimes$ Life sciences $\quad \square$ Behavioural \& social sciences $\quad \square$ Ecological, evolutionary \& environmental sciences

For a reference copy of the document with all sections, see nature.com/documents/nr-reporting-summary-flat.pdf

\section{Life sciences study design}

All studies must disclose on these points even when the disclosure is negative.

Sample size No statistical method was used to predetermine sample size. For in vivo experiments, based on preliminary studies that determined experimental variation in survival following infection and mAb treatment, we have performed power calculations and determined that at least $\mathrm{n}=6$ mice/group is sufficient to detect differences among experimental groups (powered at $80 \%$ for $5 \%$ significance level; survival assessed by log-rank (Mantel-Cox) test).

Data exclusions No data were excluded

Replication The reported findings have been confirmed in large groups of mice/experimental group in multiple ( $>2$ ) experiments. The enhanced protective activity of the Fc engineered variants was confirmed in multiple experiments using a variety of mAbs that target different epitopes on HA and NA. Also, two different challenge virus strains have been used (PR8 and Neth/09) to minimize strain-specific effects.

Randomization Mice were randomized based on age and weight. Prior to treatment, we ensured that the mean weight and age were comparable among the various treatment groups. No randomization was used for the in vitro experiments, as it is not applicable to the study design.

Blinding Blinding is not relevant for this study, as this is an observational (not case-control) study. Whenever possible (not always, due to limited access of lab staff in BSL-2 animal facilities), the treatment groups were blinded to the person involved in monitoring mouse survival and weight upon challenge (applicable to data presented in Figures 1d and 4).

\section{Reporting for specific materials, systems and methods}

We require information from authors about some types of materials, experimental systems and methods used in many studies. Here, indicate whether each material, system or method listed is relevant to your study. If you are not sure if a list item applies to your research, read the appropriate section before selecting a response.

Materials \& experimental systems

n/a Involved in the study

$\square$ Antibodies

$\square \bigotimes$ Eukaryotic cell lines

Х Palaeontology

$\triangle$ Animals and other organisms

\ $\square$ Human research participants

Х $\square$ Clinical data

\section{Antibodies}

Antibodies used

\begin{tabular}{l|l}
\multicolumn{2}{l}{ Methods } \\
\hline n/a & Involved in the study \\
\hline & $\square$ ChIP-seq \\
\hline & $\bigotimes$ Flow cytometry \\
$\square$ & $\square$ MRI-based neuroimaging
\end{tabular}

The clones of the recombinant anti-influenza antibodies are reported in the manuscript and their characteristics are also described in previously published studies (relevant citations included in the manuscript). The anti-HIV Env mAb (3BNC117) used in PK studies (Extended Data Figure 4) has been characterized in previous studies (Bournazos S et al. Cell. 2014;158(6):1243-1253.).

For generating NP-anti-NP IgG immune complexes (Extended Data Figures 1e and 7j), the high affinity anti-NP (4-hydroxy-3nitrophenylacetyl) mAb 3B62 was synthesized using the previously published sequence (Allen D et al. EMBO J.

1988;7(7):1995-2001). Another anti-NP mAb (clone 3C13) described in this publication was also cloned and used as isotype control in this study.

For ELISA assays, the following antibodies were used: HRP-conjugated goat $F\left(a b^{\prime}\right) 2$ anti-human IgG $(\mathrm{H}+\mathrm{L})(\mathrm{Jackson}$ Immunoresearch, 109-036-088, Lot: 138297, 1:5000); HRP-conjugated goat F(ab')2 anti-mouse IgG (H+L)(Jackson Immunoresearch, 115-035-146, Lot: 144209, 1:5000); Biotin-conjugated goat F(ab')2 anti-human IgG (F(ab')2-specific)(Jackson Immunoresearch, 109-066-097, Lot: 127106, 5 $\mu \mathrm{g} / \mathrm{ml}$ ).

For microneutralization assays, cells were stained with biotinylated mouse anti-influenza NP (nucleoprotein)(EMD Millipore, MAB8257B, clone A1, lot: 3286064, 1:2000).

For cellular depletion studies, all monoclonal antibodies were obtained from Bioxcell. The following mAbs were used: rat Ig2b isotype control (clone LTF-2, BE0090, lot: $707119 J 1$ and 651117D1), anti-mouse CD4 (clone GK1.5, BE0003-1, lot: 689518A1), 
The following antibodies were used for flow cytometry (unless otherwise noted, all antibodies were used at 1:250 dilution): (i) for the characterization of the FcyRlla expression of the FcyRlla+ mouse strain: anti-CD11c-eFluor506 (ThermoFisher, 69-0114-82, clone N418) anti-CD11b-BrilliantViolet605 (Biolegend, 101257, clone M1/70) anti-SiglecF-SuperBright645 (ThermoFisher, 64-1702-82, clone 1RNM44N) anti-CD8ß-BrilliantViolet711 (Biolegend, 126633, clone YTS156.7.7) anti-MHCII- BrilliantViolet785 (Biolegend, 107645, clone M5/114.15.2) anti-human FcyRIla-FITC (Stemcell technologies, 60012FI, clone IV.3, used at 1:50 dilution) anti-GR-1-PerCP/Cy5.5 (ThermoFisher, 45-5931-80, clone RB6-8C5) anti-CD3-PE anti-CD3-PE (Biolegend, 100206, clone 17A2) anti-CD103-PE/eFluor610 (ThermoFisher, 61-1031-82, clone 2E7) anti-NK1.1-PE/Cy7 (ThermoFisher, 25-5941-82, clone PK136) anti-CD4-AlexaFluor647 (Biolegend, 100530, clone RM4-5) anti-CD19-AlexaFluor700 (Biolegend, 115527, clone 6D5) Mouse IgG2b kappa isotype control - FITC (ThermoFisher, 11-4732-42, clone eBMG2b, used at 1:50 dilution)

(ii) for the evaluation of FcyR expression on innate effector leukocytes: anti-CD11c-eFluor506 (ThermoFisher, 69-0114-82, clone N418) anti-human FcyRI-BrilliantViolet605 (Biolegend, 305034, clone 10.1, used at 1:100 dilution) anti-SiglecF- SuperBright645 (ThermoFisher, 64-1702-82, clone 1RNM44N) anti-Ly6G-BrilliantViolet711 (Biolegend, 127643, clone 1A8) anti-CD11b-BrilliantViolet785 (Biolegend, 101243, clone M1/70) anti-human FcyRIla-FITC (Stemcell technologies, 60012FI, clone IV.3, used at 1:50 dilution) anti-Ly6C-PerCP/Cy5.5 (ThermoFisher, 45-5932-82, clone HK1.4) anti-human FcyRIIla/b-PE (ThermoFisher, MHCD1604, clone 3G8, used at 1:100 dilution) anti-CD103-PE/eFluor610 (ThermoFisher, 61-1031-82, clone 2E7) anti-NK1.1-PE/Cy7 (ThermoFisher, 25-5941-82, clone PK136) anti-human FcyRIlb-Dylight650; (clone 2B6, produced in house and conjugated with Dylight650 kit, used at $10 \mu \mathrm{g} / \mathrm{ml}$ ) Mouse IgG1 isotype control-Dylight650 (ThermoFisher, MA1-191-D650, clone MOPC-21, used at 10 g/ml) Mouse IgG2b kappa isotype control - FITC (ThermoFisher, 11-4732-42, clone eBMG2b, used at 1:50 dilution) Mouse IgG1 kappa isotype control-PE (ThermoFisher, 12-4714-82, clone P3.6.2.8.1, used at 1:100 dilution) Mouse IgG1 kappa isotype control-Brilliant Violet605 (Biolegend, 400162, clone MOPC-21, used at 1:100 dilution)

(iii) for the evaluation of FcyR expression and activation status of DCs: anti-CD11c-eFluor506 (ThermoFisher, 69-0114-82, clone N418) anti-human FcyRI-BrilliantViolet605 (Biolegend, 305034, clone 10.1, used at 1:100 dilution) anti-Siglec F- SuperBright645 (ThermoFisher, 64-1702-82, clone 1RNM44N) anti-CD80-BrilliantViolet711 (Biolegend, 104743, clone 16-10A1) anti-CD11b-BrilliantViolet785 (Biolegend, 101243, clone M1/70) anti-human FcyRIla-FITC (Stemcell technologies, 60012FI, clone IV.3, used at 1:50 dilution) anti-GR-1-PerCP/Cy5.5 (ThermoFisher, 45-5931-80, clone RB6-8C5) anti-human FcyRIIla/b-PE (ThermoFisher, MHCD1604, clone 3G8, used at 1:100 dilution) anti-CD103-PE/eFluor610 (ThermoFisher, 61-1031-82, clone 2E7) anti-CD86-PE/Cy7 (ThermoFisher, 25-0862-82, clone GL1) anti-human FcyRIIb-Dylight650 (clone 2B6, produced in house and conjugated with Dylight650 kit, used at $10 \mu \mathrm{g} / \mathrm{ml}$ ) anti-MHCll-AlexaFluor700 (eBioscience, 56-5321-82, clone M5/114.15.2) Mouse IgG1 isotype control-Dylight650 (ThermoFisher, MA1-191-D650, clone MOPC-21, used at $10 \mu \mathrm{g} / \mathrm{ml}$ ) Mouse IgG2b kappa isotype control - FITC (ThermoFisher, 11-4732-42, clone eBMG2b, used at 1:50 dilution) Mouse IgG1 kappa isotype control-PE (ThermoFisher, 12-4714-82, clone P3.6.2.8.1, used at 1:100 dilution) Mouse IgG1 kappa isotype control-Brilliant Violet605 (Biolegend, 400162, clone MOPC-21, used at 1:100 dilution)

(iv) for the evaluation of CD8 or CD4 depletion: anti-CD3e-eFluor506 (ThermoFisher, 69-0032-82, clone 17A2) anti-CD19-BrilliantViolet605 (Biolegend, 115540, clone 6D5) anti-CD8b-BrilliantViolet711 (Biolegend, 126633, clone YTS156.7.7) anti-CD11b-PE (Biolegend, 101208, clone M1/70) anti-NK1.1-PE/Cy7 (ThermoFisher, 25-5941-82, clone PK136) anti-CD4-FITC, (ThermoFisher, 11-0043-82, clone RM4-4) anti-GR-1-PerCP/Cy5.5 (ThermoFisher, 45-5931-80, clone RB6-8C5) anti-NKp46-eFluor660 (ThermoFisher, 50-3351-82, clone 29A1.4) anti-B220-APC/eFluor780 (ThermoFisher, 47-0452-82, clone RA3-6B2)

(v) for the evaluation of neutrophil depletion by anti-Gr-1 mAb: anti-CD11c-eFluor506 (ThermoFisher, 69-0114-82, clone N418) anti-CD19-BrilliantViolet605 (Biolegend, 115540, clone 6D5) anti-SiglecF- SuperBright645 (ThermoFisher, 64-1702-82, clone 1RNM44N) anti-Ly6G-BrilliantViolet711 (Biolegend, 127643, clone 1A8) anti-CD11b-BrilliantViolet785 (Biolegend, 101243, clone M1/70) anti-CD8a-FITC, (ThermoFisher, MA5-16759, clone KT15) anti-Ly6C-PerCP/Cy5.5 (ThermoFisher, 45-5932-82, clone HK1.4) anti-CD3-PE (Biolegend, 100206, clone 17A2) anti-NK1.1-PE/Cy7 (ThermoFisher, 25-5941-82, clone PK136) anti-CD4-AlexaFluor647 (Biolegend, 100530, clone RM4-5) 
(vi) for the assessment of FcyR expression on T cells:

anti-B220-BrilliantViolet421 (Biolegend, 103240, clone RA3-6B2)

anti-CD3-BrilliantViolet510 (Biolegend, 100353, clone 145-2C11)

anti-human FcyRI (clone 10.1)-BrilliantViolet 605 (Biolegend, 305034, clone 10.1, used at 1:100 dilution)

anti-CD8-BrilliantViolet711 (Biolegend, 100748, clone 53-6.7)

anti-CD4-BrilliantViolet785 (Biolegend, 100453, clone GK1.5)

anti-human FcyRIla (clone IV.3)-FITC (Stemcell technologies, 60012FI, clone IV.3, used at 1:50 dilution)

anti-NK1.1-PerCP/Cy5.5 (Biolegend, 108728, clone PK136)

anti-human FcyRIIIa/b (clone 3G8)-PE (ThermoFisher, MHCD1604, clone 3G8, used at 1:100 dilution)

anti-CD11b-PE/Cy7 (Biolegend, 101216, clone M1/70)

anti-human FcyRIIb (clone 2B6)-Dylight650 (clone 2B6, produced in house and conjugated with Dylight $650 \mathrm{kit}$, used at $10 \mu \mathrm{g} / \mathrm{ml}$ ) anti-GR-1-AlexaFluor700 (Biolegend, 108422, clone RB6-8C5)

Mouse IgG1 isotype control-Dylight650 (ThermoFisher, MA1-191-D650, clone MOPC-21, used at $10 \mu \mathrm{g} / \mathrm{ml}$ )

Mouse IgG2b kappa isotype control - FITC (ThermoFisher, 11-4732-42, clone eBMG2b, used at 1:50 dilution)

Mouse IgG1 kappa isotype control-PE (ThermoFisher, 12-4714-82, clone P3.6.2.8.1, used at 1:100 dilution)

Mouse IgG1 kappa isotype control-Brilliant Violet605 (Biolegend, 400162, clone MOPC-21, used at 1:100 dilution)

(vii) for the characterization of DC populations following mAb treatment:

anti-CD103-FITC (Biolegend, 121420, clone 2E7)

anti-Ly6C-PerCP/Cy5.5 (ThermoFisher, 45-5932-82, clone HK1.4)

anti-NK1.1-AlexaFluor647 (Biolegend, 108720, clone PK136)

anti-CD45-AlexaFluor700 (ThermoFisher, 56-0451-82, clone 30-F11)

anti-CD11c-eFluor506 (ThermoFisher, 69-0114-82, clone N418)

anti-CD86-BrilliantViolet605 (Biolegend, 105037, clone GL-1)

anti-SiglecF- SuperBright645 (ThermoFisher, 64-1702-82, clone 1RNM44N)

anti-Ly6G-BrilliantViolet711 (Biolegend, 127643, clone 1A8)

anti-CD11b-BrilliantViolet785 (Biolegend, 101243, clone M1/70)

anti-CD40-PE (Biolegend, 124610, clone 3/23)

anti-MHCII-PE/eFluor610 (ThermoFisher, 61-5321-82, clone M5/114.15.2)

anti-CD80-PE/Cy7 (Biolegend, 104734, clone 16-10A1)

(viii) for the characterization of T-cell populations following mAb treatment:

anti-CD4-AlexaFluor488 (Biolegend, 100423, clone GK1.5)

anti-CD3e-PerCP/Cy5.5 (Biolegend, 100218, clone 17A2)

anti-NK1.1-AlexaFluor 647 (Biolegend, 108720, clone PK136)

anti-CD45-AlexaFluor 700 (ThermoFisher, 56-0451-82, clone 30-F11)

anti-CD44-BrilliantViolet 421 (Biolegend, 103040, clone IM7)

anti-CD62L-BrilliantViolet 510 (Biolegend, 104441, clone MEL-14)

anti-CD25-BrilliantViolet 605 (Biolegend, 102036, clone PC61)

anti-CD27-BrilliantViolet 650 (Biolegend, 124233, clone LG.3A10)

anti-CD8-BrilliantViolet 711 (Biolegend, 100748, clone 53-6.7)

anti-CD11a-PE (Biolegend, 153104, clone 121/7)

anti-CCR7-PE/eFluor 610 (ThermoFisher, 61-1971-82, clone 4B12)

anti-CD69-PE/Cy7 (Biolegend, 104512, clone H1.2F3)

(ix) for the assessment of FcR expression on human T cells (all antibodies were used at 1:100 dilution unless otherwise stated): anti-CD14-BrilliantViolet510 (Biolegend, 301841, clone M5E2)

anti-CD19-BrilliantViolet510 (Biolegend, 302242, clone HIB19)

anti-human FcyRI-BrilliantViolet605 (Biolegend, 305034, clone 10.1)

anti-CD8-BrilliantViolet650 (Biolegend, 344729, clone SK1)

anti-CD3-BrilliantViolet711 (Biolegend, 300464, clone UCHT1)

anti-CD11b-BrilliantViolet785 (Biolegend, 101243, clone M1/70)

anti-human FcyRIIa-FITC (Stemcell technologies, 60012FI, clone IV.3, used at 1:50 dilution)

anti-CD4-PerCP-Cy5.5 (Biolegend, 317427, clone OKT4)

anti-human FcyRIIla/b-PE (ThermoFisher, MHCD1604, clone 3G8)

anti-CD56-PE/Cy7 (Biolegend, 362509, clone 5.1H11)

anti-human FcyRIIb-Dylight650 (clone 2B6, produced in house and conjugated with Dylight650 kit, used at $10 \mu \mathrm{g} / \mathrm{ml}$ )

anti-CD41-AlexaFluor700 (Biolegend, 303737, clone HIP8)

anti-CD42b-AlexaFluor700 (Biolegend, 303928, clone HIP1)

Mouse IgG1 isotype control-Dylight650 (ThermoFisher, MA1-191-D650, clone MOPC-21, used at $10 \mu \mathrm{g} / \mathrm{ml}$ )

Mouse IgG2b kappa isotype control - FITC (ThermoFisher, 11-4732-42, clone eBMG2b, used at 1:50 dilution)

Mouse IgG1 kappa isotype control-PE (ThermoFisher, 12-4714-82, clone P3.6.2.8.1)

Mouse IgG1 kappa isotype control-Brilliant Violet605 (Biolegend, 400162, clone MOPC-21)

(x) for the evaluation of ex vivo DC maturation of human monocyte-derived DCs (all antibodies were used at 1:100 dilution unless otherwise stated):

anti-DC-SIGN-BrilliantViolet421 (Biolegend, 330118, clone 9E9A8)

anti-CD40-BrilliantViolet510 (Biolegend, 334330, clone 5C3)

anti-human FcyRI-BrilliantViolet605 (Biolegend, 305034, clone 10.1)

anti-CD11b-BrilliantViolet650 (Biolegend, 301336 clone ICRF44)

anti-CD86-BrilliantViolet711 (Biolegend, 305440 clone IT2.2)

anti-human FcyRIla-FITC (Stemcell technologies, 60012FI, clone IV.3, used at 1:50 dilution)

anti-CD11c-PerCP-Cy5.5 (Biolegend, 301624, clone 3.9) 
anti-human FcyRIIIa/b-PE (ThermoFisher, MHCD1604, clone 3G8)

anti-CD80-PE/Cy7 (Biolegend, 305218, clone 2D10)

anti-human FcyRllb-Dylight650 (clone 2B6, produced in house and conjugated with Dylight650 kit, used at $10 \mu \mathrm{g} / \mathrm{ml}$ ) anti-HLA-DR-AlexaFluor700 (Biolegend, 327014, clone LN3)

anti-CD83-APC/Cy7 (Biolegend, 305330, clone HB15e)

Validation

For recombinant anti-influenza mAbs, their antigenic specificity, $\mathrm{HAl}$, and neutralization activity were assessed in in vitro assays (data presented in the manuscript Extended Data Figure 3) to ensure that changes in the Fc domain have no impact of their Fabmediated activities. Purity of recombinant proteins was assessed by SDS-PAGE followed by SafeStain blue staining (ThermoFisher). All antibody preparations were $>90 \%$ pure and endotoxin levels were $<0.05 \mathrm{EU} / \mathrm{mg}$, as determined by the Limulus Amebocyte Lysate (LAL) assay.

For commercially available antibodies, validation has been performed by the manufacturer and corresponsing certificates of analysis are available at the manufacturer's website. Reactivity of primary antibodies used in flow cytometry assays was validated by the manufacturer in immunocytochemistry and frozen immunohistochemistry. Antibodies used for microneutralization assay and ELISA were validated by the manufacturer for cross-reactivity to other influenza strains (for anti-NP mAb) or to immunoglobulins from other species. For antibodies used for in vivo cell depletion, antibody preparations have been validated by he manufacturer for specificity, purity, and endotoxin content.

\section{Eukaryotic cell lines}

Policy information about cell lines

Cell line source(s)

ATCC for MDCK, ThermoFisher for Expi293F and Expi-CHO cells, and Promega for NFAT reporter assays

Authentication

Cell lines were not authenticated after purchase. Cell lines obtained from Promega were autheticated by the manufacturer by STR analysis.

Mycoplasma contamination

All cell lines have been tested by the manufacturer and were tested negative for mycoplasma contamination. Certificates of analysis are available in manufacturer's website. All cell lines were maintained for $<10$ passages to minimize mycoplasma contamination. Media and supplements (FBS) were also all mycoplasma-free (per certificate of analysis).

Commonly misidentified lines (See ICLAC register)

No commonly misidentified cell lines were used in this study.

\section{Animals and other organisms}

Policy information about studies involving animals; ARRIVE guidelines recommended for reporting animal research

Laboratory animals

C57BI/6 were purchased from The Jackson Laboratory. FcyR humanized mice (FcyRanull, hFcyRl+, FcyRIlaR131+, FcyRIIb+, FcyRIIlaF158+, and FcyRIIIb+) were generated in the C57BI/6 background and extensively characterized in previous studies. FcRn humanized mice (B6.Cg-Fcgrttm1Dcr Tg(FCGRT)32Dcr/DcrJ) were purchased from The Jackson Laboratory and are deficient in mouse FcRn and express human FcRn as transgene. FcyR/FcRn humanized mice were generated by crossing the $F_{c y} R$ humanized strain to the FcRn humanized mice. FcyRlla+ mice were generated on a mouse FcyR KO background (mouse FcR null; C57BI/6). For in vivo experiments, female or male, 6-12 wk old mice were used. Mice were housed at a controlled ambient temperature environment with 12 -hour light/dark cycle.

Wild animals

No wild animals were used in this study.

Field-collected samples

No field-collected samples were used in this study.

Ethics oversight

All in vivo experiments were performed in compliance with federal laws and institutional guidelines and have been approved by the Rockefeller University Institutional Animal Care and Use Committee.

Note that full information on the approval of the study protocol must also be provided in the manuscript.

\section{Flow Cytometry}

Plots

Confirm that:

\The axis labels state the marker and fluorochrome used (e.g. CD4-FITC).

$\bigotimes$ The axis scales are clearly visible. Include numbers along axes only for bottom left plot of group (a 'group' is an analysis of identical markers).

$\bigotimes$ All plots are contour plots with outliers or pseudocolor plots.

$\bigotimes$ A numerical value for number of cells or percentage (with statistics) is provided.

Methodology

Sample preparation

Mice (strains described in animal section) were euthanized and lungs were perfused by injection of PBS (containing $10 \mathrm{U} / \mathrm{ml}$ 
Sample preparation

Instrument

Software

Cell population abundance

Gating strategy heparin) into the right cardiac ventricle. Lungs were excised and homogenized using the gentleMACS dissociator (Mouse lung dissociation kit (Miltenyi)), according to the manufacturer's recommendations. Mouse blood was collected from the retro-orbital venous plexus. Spleens were excised from mice and homogenized by mechanical shearing. Following RBC lysis (RBC lysis buffer; Biolegend), single cell suspensions were labelled with the LIVE/DEAD Fixable Near-IR (ThermoFisher) and resuspended in PBS containing $0.5 \%(\mathrm{w} / \mathrm{v}) \mathrm{BSA}$ and $5 \mathrm{mM}$ EDTA. Cells were labelled with mixtures of fluorescently labelled antibodies incubated at $40 \mathrm{C}$ for $15 \mathrm{~min}$ and washed twice with PBS containing $0.5 \%$ (w/v) BSA and 5 mM EDTA prior to analysis. Human PBMCs and monocyte-derived DCs were prepared for flow cytometry analysis as described above.

OA29009 Attune NxT Acoustic Focusing Cytometer (Lasers: BRV6Y)

For data acquisition, Attune NxT flow cytometry software v3.1.2 was used. For data analysis, FlowJo v10.6.2 with the following plugins: Downsample v3.2, UMAP v2.2, and X-shift v1.3 was used.

No sorting performed

The gating strategies are presented in Extended Data Figures 5-9. Single cells were identified in FSC-A vs. FSC-H plots and live cells based on LIVE/DEAD staining vs. FSC-A.

For characterization of FCRIla expression on lung-resident leukocytes, live single cells are plotted as NK1.1 vs. CD11b and NK cells were identified as NK1.1+/CD11b-. From the NK1.1- gate, cells are plotted as CD11c vs. CD11b. From CD11b+/CD11c- cells, neutrophils are defined as Gr-1+/SSChigh and monocytes as SSClo/Gr-1 int or negative. From the CD11C+ gate, alveolar macrophages are identified as SiglecF+/CD11b-, whereas SiglecF- events are further gated and MHCII+ events are selected. $\mathrm{MHCll}+$ cells are divided into cDC1 and cDC2 based on CD103 and CD11b expression (cDC1: CD103+/CD11b-; cDC2: CD103-/ $\mathrm{CD} 11 \mathrm{~b}+$ ). From the CD11b-/CD11c- gate, B cells are identified as CD19+/MHCll+, whereas CD19- events are gated based on CD3. CD3+ events are plotted as CD8 vs. CD4 to identify CD8 (CD8+/CD4-) and CD4 (CD4+/CD8-) T cells. The gating strategy and boundaries are presented in Extended Data Figure 5 a.

For experiments assessing FcR expression in the lungs of influenza-infected mice, NK cells are identified as NK1.1+/CD11b-; from the NK1.1- gate, cells are plotted as CD11c vs. CD11b. From CD11b+/CD11c-cells, neutrophils are defined as Ly6G+/Ly6Cint, Ly6Chi monocytes as Ly6Chi/Ly6G-, and Ly6Cint monocytes as Ly6Cint/Ly6G- events. From the Ly6C-Ly6G- gate, eosinophils are defined as SSChi/SiglecF+ cells. From the CD11c+ gate, alveolar macrophages are defined as CD11b-/Siglec F+ cells. The gating boundaries are presented in Extended Data Figure 6a.

Lung-resident DC populations are identified based on the following gating strategy: CD11c+ cells are plotted as Siglec F vs. CD11b. From the Siglec F- gate, MHCII+ cells are selected and plotted as CD103 vs. CD11b. CDC1 are identified as CD103+/ CD11b-cells. CD11b+/CD103- cells are further separated into Gr-1 positive and negative cells, representing tipDCs (Gr-1+) and CDC2 (Gr-1-), respectively. The gating strategy and boundaries for identifying DC populations are presented in Extended Data Figure 7.

For analysis of FcR expression on human T cells, T cells are defined as CD41/CD42-/CD11b- events, which are further gated to select CD56-/CD19-/CD14- cells. From this gate, CD3+ cells are divided into CD4 (CD4+/CD8-) and CD8 (CD8+/CD4-) T cells. Monocytes and B cells are identified from single cells based on CD19/CD14/CD11b staining: B cells as CD11b-/CD19+ and monocytes as CD11b+/CD14+. Detailed gating strategy is presented in Extended Data Figure 9a.

For analysis of FCR expression on mouse blood T cells, single cell lymphocytes (identified based on FSC-A vs. FSC-H and FSC-ASSC-A plots) are gated as CD11b-/Gr-1- cells, which are further gated as B220-/NK1.1-. The resulting population is then gated to select CD3 T cells, which are plotted as CD8 vs. CD4 to identify CD8 (CD8+/CD4-) and CD4 (CD4+/CD8-) T cells. CD11b+ and B220 + cells are also selected from the single cell gate and used as controls for FcR staining. The gating strategy and boundaries are presented in Extended Data Figure 9c.

For analysis of FcR expression on mouse spleen and lung T cells, live single cells (identified based on FSC-A vs. FSC-H and Live/ dead staining) are gated as CD11b vs. B220 to identify CD11b+ and B220+ cells. To identify T cells, NK1.1-/Gr-1- cells are gated as CD11b vs. B220. CD11b-/B220- events are gated to select CD3+ T cells. CD3+ events are plotted as CD8 vs. CD4 to identify CD8 (CD8+/CD4-) and CD4 (CD4+/CD8-) T cells. The gating strategy and boundaries are presented in Extended Data Figure 9e.

Tick this box to confirm that a figure exemplifying the gating strategy is provided in the Supplementary Information. 